\title{
STING directly activates autophagy to tune the innate immune response
}

\author{
Dong $\mathrm{Liu}^{1,2} \cdot \mathrm{Hao} \mathrm{Wu}^{1} \cdot$ Chenguang $\mathrm{Wang}^{3} \cdot$ Yanjun $\mathrm{Li}^{1} \cdot$ Huabin Tian ${ }^{2,4} \cdot$ Sami Siraj ${ }^{1,5} \cdot$ Sheikh Arslan Sehgal ${ }^{1,2} \cdot$ \\ Xiaohui Wang ${ }^{1} \cdot$ Jun Wang $^{1} \cdot$ Yingli Shang $^{6} \cdot$ Zhengfan Jiang $^{3} \cdot$ Lei Liu $^{1} \cdot$ Quan Chen $^{1,2,7}$
}

Received: 11 April 2018 / Revised: 28 September 2018 / Accepted: 7 November 2018 / Published online: 19 December 2018

(c) ADMC Associazione Differenziamento e Morte Cellulare 2018

\begin{abstract}
STING (stimulator of interferon genes) is a central molecule that binds to cyclic dinucleotides produced by the cyclic GMPAMP synthase (cGAS) to activate innate immunity against microbial infection. Here we report that STING harbors classic LC-3 interacting regions (LIRs) and mediates autophagy through its direct interaction with LC3. We observed that poly(dA: dT), cGAMP, and HSV-1 induced STING-dependent autophagy and degradation of STING immediately after TBK1 activation. STING induces non-canonical autophagy that is dependent on ATG5, whereas other autophagy regulators such as Beclin1, Atg9a, ULK1, and p62 are dispensable. LIR mutants of STING abolished its interaction with LC3 and its activation of autophagy. Also, mutants that abolish STING dimerization and cGAMP-binding diminished the STING-LC3 interaction and subsequent autophagy, suggesting that STING activation is indispensable for autophagy induction. Our results thus uncover dual functions of STING in activating the immune response and autophagy, and suggest that STING is involved in ensuring a measured innate immune response.
\end{abstract}

Edited by M. Piacentini

Supplementary information The online version of this article (https:// doi.org/10.1038/s41418-018-0251-z) contains supplementary material, which is available to authorized users.

$\triangle$ Lei Liu

liulei@ioz.ac.cn

$\triangle$ Quan Chen

chenq@ioz.ac.cn

1 State Key Laboratory of Membrane Biology, Institute of Zoology, Chinese Academy of Sciences, Beijing, China

2 University of Chinese Academy of Sciences, Beijing, China

3 College of Life Sciences, Peking University, Beijing, China

4 Key Laboratory of Infection and Immunity, Institute of Biophysics, Chinese Academy of Sciences, Beijing, China

5 Institute of Basic Medical Sciences, Khyber Medical University, Peshawar, Pakistan

6 Shandong Provincial Key Laboratory of Animal Biotechnology and Disease Control and Prevention, College of Veterinary Medicine, Shandong Agricultural University, Tai' an, China

7 College of Life Sciences, Nankai University, Tianjin, China

\section{Introduction}

Innate immunity is the first barrier for defending host cells against invading microbial pathogens, which are recognized by diverse pattern recognition receptors (PRRs). Pathogenderived DNA is sensed by cytosolic DNA sensors such as cyclic GMP-AMP synthase (cGAS), which directly binds to DNA and further catalyzes the synthesis of cyclic GMPAMP (cGAMP) from ATP and GTP [1, 2]. cGAMP serves as a second messenger and forms a complex with the endoplasmic reticulum (ER)-resident protein STING (stimulator of interferon genes; also known as MITA, ERIS or MPYS) [3-6], leading to its conformational change and self-activation. Subsequently, STING traffics from the ER through the Golgi apparatus and ultimately to the perinuclear compartments [7, 8]. During this process, TBK1 (TANK-binding kinase 1), a pivotal kinase of innate immunity cascades, is recruited to STING and activated, leading to the subsequent phosphorylation of transcription factor IRF3 (interferon regulatory factor 3). The phosphorylated IRF3 further dimerizes and translocates into the nucleus, initiating transcription of the gene encoding type I interferon (IFN) [9]. In addition, STING also triggers a series of signaling cascades to induce transcription factor 
$\mathrm{NF}-\kappa \mathrm{B}$ to enter the nucleus, resulting in the expression of inflammatory cytokines such as TNF, IL-1 $\beta$, and IL-6 [10].

The human STING protein has 379 amino acids and comprises three functional domains. The first domain contains four N-terminal transmembrane helices (TM1-TM4) that anchor STING to the ER; the second is a central c-diGMP-binding domain (CBD) that binds the bacterial second messenger cyclic di-GMP and mediates homodimerization for activation of STING; and the third domain is a cytoplasmic C-terminal tail (CTT) that is responsible for TBK1 and IRF3 activation [11]. As a pivotal regulator of innate immunity cascades, STING activity must be tightly regulated to ensure a measured response, and STING-mediated immune activation must also be terminated in a timely manner to prevent excessive STING activation. Protein post-translational modifications including phosphorylation and ubiquitination have been reported to fine-tune STINGmediated innate immunity responses. It has been found that TBK1 phosphorylates STING at several serine residues and positively regulates STING signaling cascades [6, 12, 13]. A series of E3 ubiquitin ligases, such as TRIM56, TRIM32, RNF5, and RNF26, participate in regulating STING activation by augmenting or attenuating its polyubiquitination [14-17]

Autophagy is a fundamental cellular process in eukaryotes that involves sequestration of cytosolic constituents within double membrane-bound autophagosomes which are subsequently fused with endolysosomal vesicles, leading to degradation and recycling of the sequestered substrates. Autophagy is thus a highly conserved intracellular degradation process for eliminating damaged organelles, protein aggregates and invading pathogens in eukaryotes [18]. Emerging evidence shows that autophagy functions in multiple biological processes including innate immune responses, inflammation, and Wnt signaling [19-22]. Conversely, a number of innate immunity-related proteins have been found to control autophagy. TBK1 has been reported to promote selective autophagy by phosphorylating autophagy receptors such as p62, OPTN [23-26]. STING participates in regulation of autophagy flux, leading to cell death in breast cancer cells [27]. Recently, STING was found to sense live gram-positive bacteria and to trigger ER-phagy [28]. In addition, Atg9a was reported to limit dsDNAinduced innate immune responses by controlling the translocation of STING [7]. Serine/threonine UNC-51-like kinase (ULK1/ATG1) has been reported to phosphorylate STING at serine 366 (S366) and promote subsequent STING degradation, which serves as a negative-feedback control of STING activity and prevents the persistent activation of innate immune responses [29]. Also, p62/ SQSTM1 mediates STING degradation and attenuation of the response [30]. These studies highlight the interplay between autophagy and STING-mediated innate immunity.
However, the precise mechanism by which STING regulates autophagy remains unclear. In this study, we found that STING is a potential autophagy receptor and directly interacts with LC3 via its LIR motifs to mediate autophagy and its own autophagic degradation to tune the innate immune responses.

\section{Results}

\section{Poly(dA:dT) and cGAMP induce STING-dependent autophagy and its autophagic degradation}

STING functions as a direct sensor of cyclic dinucleotides (CDNs) to activate innate immunity signaling pathways. As expected, poly (dA:dT) and cGAMP, two activators of the STING pathway, were able to induce TBK1 phosphorylation, a commonly used indicator of STING activation, in MEF cells at $1 \mathrm{~h}$ and $2 \mathrm{~h}$, respectively (Supplementary Fig. 1a and b). Interestingly, poly(dA:dT) and cGAMP promoted LC3-II conversion, a biochemical hallmark of autophagy, at 2 or $8 \mathrm{~h}$ (Supplementary Fig. 1a and b). However, this conversion was completely blocked in Sting knockout cells (Fig. 1a, d). Immunofluorescence analysis showed that poly(dA:dT) and cGAMP induced the formation of LC3 puncta, and this was largely abrogated in Sting knockout MEF cells (Fig. 1b, c, e and f). We also noticed that poly(dA:dT) and cGAMP treatments significantly reduced the protein levels of STING as early as 1 and $4 \mathrm{~h}$ after TBK1 activation (Supplementary Fig. 1a and b, Fig. 1a, d). As shown in Fig. 1g, h, poly(dA:dT)-induced and cGAMP-induced STING degradation were blocked by treatment with the lysosome inhibitor chloroquine (CQ), while the proteasome inhibitor MG132 was less effective in preventing STING degradation (Fig. 1i and Supplementary Fig. 1c). Collectively, these results provide evidence that activation of STING signaling is followed by autophagy induction and the autophagic degradation of STING itself.

\section{STING induces ATG5-dependent autophagy}

We next asked how STING triggers autophagy. The ectopic expression of STING markedly increased the levels of LC3 and p62 puncta in both HeLa and wild-type MEF cells (Fig. 2a, b). This was accompanied by p62 degradation and LC3-II conversion, whereas there was no obvious changes in the mitochondrial protein ATPB or the ER protein Calnexin (Fig. 2c), which indicates that ectopic expression of STING has little, if any, effect on ER-phagy. Similarly, STING augmented the formation of LC3 and p62 puncta in MCF-7 and HepG2 cells (Supplementary Fig. 2a-d). EM analysis also revealed an increased number of autophagic 

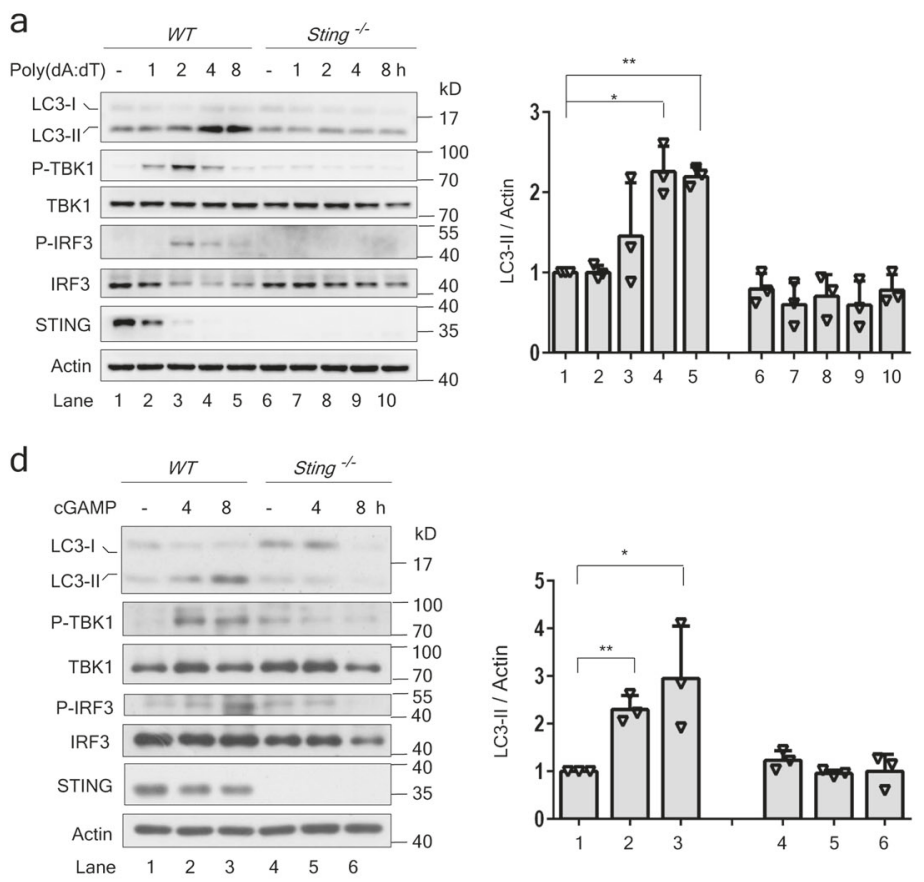

g

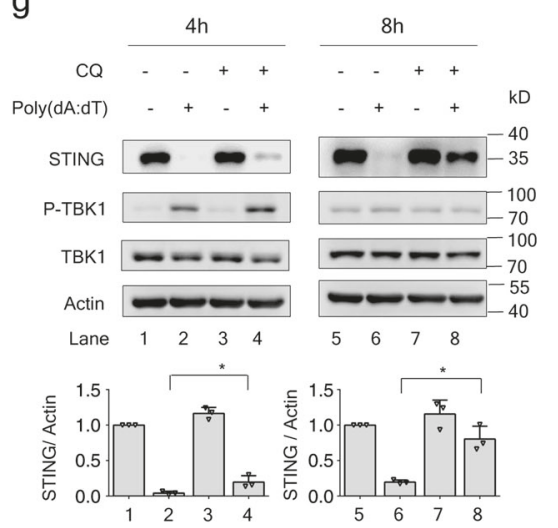

h

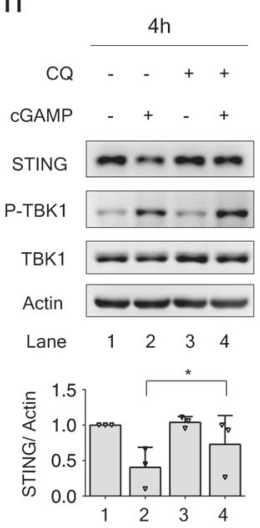

Fig. 1 Poly(dA:dT) and cGAMP induce STING-dependent autophagy and degradation of STING. a Immunoblot analysis of LC3-II expression levels in Sting wild-type and knockout MEF cells stimulated by poly(dA:dT) at a final concentration of $1 \mu \mathrm{g} / \mathrm{ml}$. Quantification of LC3-II expression levels in $\mathbf{a}$ is shown in the right panel (mean $\pm \mathrm{s}$. d.; from three independent experiments). ${ }^{*} P<0.05,{ }^{* *} P<0.01$. b Sting wild-type and knockout MEFs were transfected with a plasmid expressing GFP-LC3 and stimulated with or without poly(dA:dT) for $8 \mathrm{~h}$. Images were then captured by confocal microscopy. Scale bar: 10 $\mu \mathrm{m}$. $\mathbf{c}$ The numbers of GFP-LC3 dots per cell in $\mathbf{b}$ were quantified (mean \pm s.d.; $n>100$ cells from three independent experiments). ${ }^{* * *} P$ $<0.001$. ns, not significant. d cGAMP was used to stimulate Sting wild-type and knockout MEFs for the indicated time, and cell lysates were then subjected to western blotting analysis of LC3-II conversion. Quantification of LC3-II expression levels in $\mathbf{d}$ is shown in the right panel (mean \pm s.d.; from three independent experiments). ${ }^{*} P<0.05$, ${ }^{* *} P<0.01$. e Sting wild-type and knockout MEFs were transfected with the GFP-LC3 plasmid and then treated with or without cGAMP at a final concentration of $5 \mu \mathrm{g} / \mathrm{ml}$ for $8 \mathrm{~h}$. Formation of LC3 puncta was

vesicles in cells transfected with wild-type Flag-STING (Supplementary Fig. 2e). Additionally, Chloroquine enhanced the level of LC3-II, suggesting that STING alone

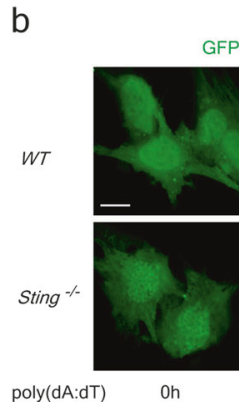

GFP-LC3
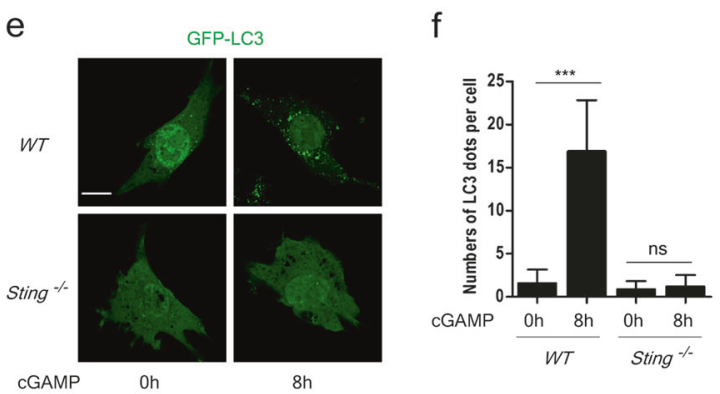

C
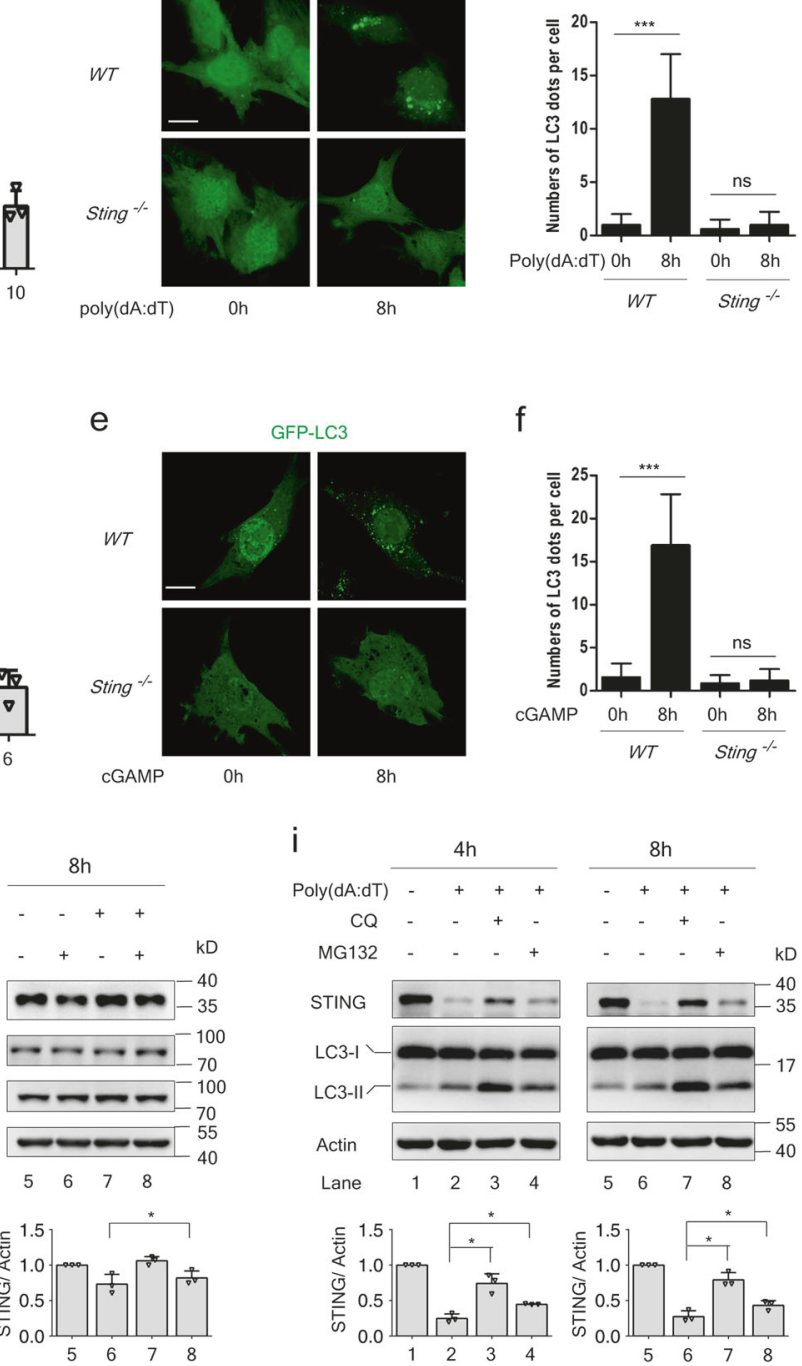

then examined by immunofluorescence microscopy. Scale bar: $10 \mu \mathrm{m}$. f Quantification of the numbers of LC3 dots per cell (mean \pm s.d.; $n>$ 100 cells from three independent experiments). ${ }^{* * *} P<0.001$. ns, not significant. g Poly(dA:dT) was used to stimulate MEF cells for the indicated time without or with chloroquine (CQ) treatment. Western blotting was used to detect protein expression levels with the indicated antibodies. Quantification of STING expression levels is shown below the Fig. $1(\mathrm{~g})$ (mean \pm s.d.; from three independent experiments). ${ }^{*} P<$ 0.05. h cGAMP was used to stimulate MEF cells for the indicated time without or with chloroquine (CQ) treatment. Western blotting was used to detect protein expression levels with the indicated antibodies. Quantification of STING expression levels is shown below the Fig. 1 (h) (mean \pm s.d.; from three independent experiments). ${ }^{*} P<0.05$. i Poly(dA:dT) was used to stimulate MEF cells for the indicated time without or with chloroquine (CQ) or MG132 treatment, and the cell lysates were subjected to immunoblot analysis. Quantification of STING expression levels is shown below the Fig. 1(i) (mean \pm s.d.; from three independent experiments). ${ }^{*} P<0.05$

induces autophagic flux (Fig. 2d). Also, poly(dA:dT) stimulation greatly increased the autophagic flux in Sting wild-type but not in knockout cells (Supplementary Fig. $2 \mathrm{f}$ 
a
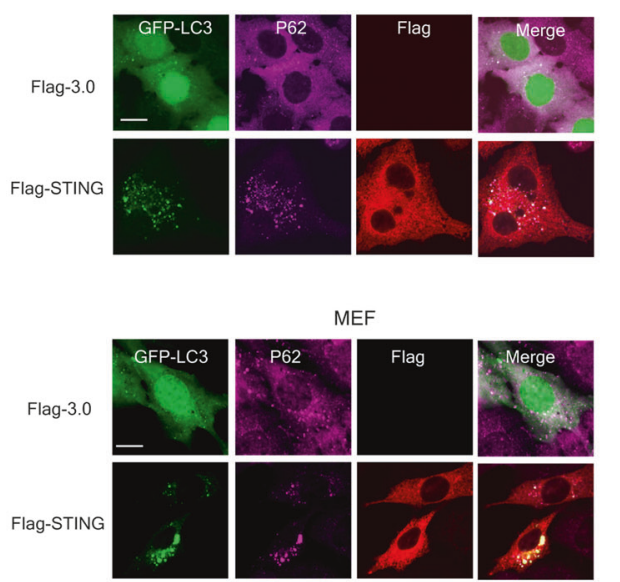

e

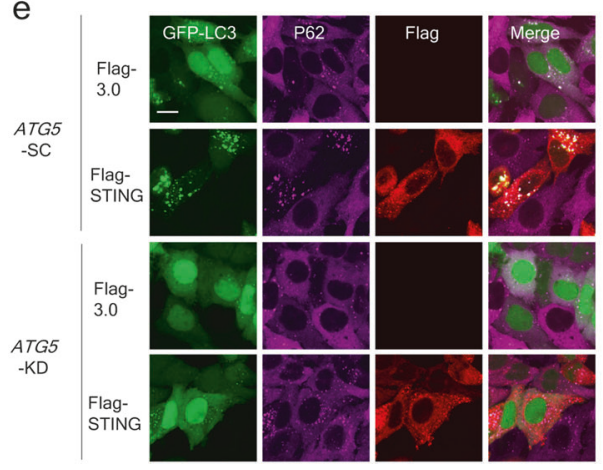

b

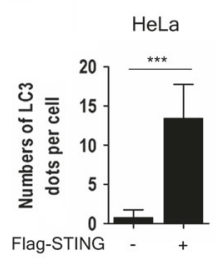

C

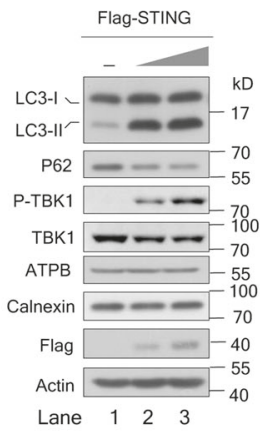

d

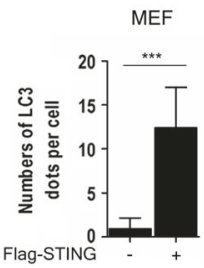

d Flag-STING $\frac{-\mathrm{CQ}}{-+\mathrm{CQ}}$
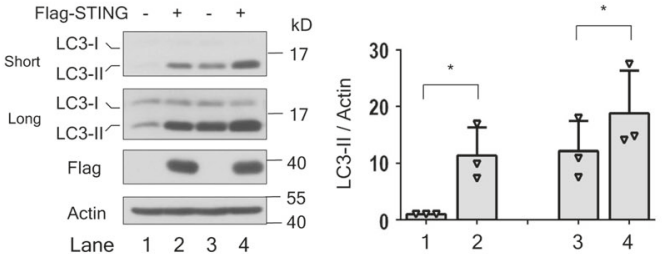

f $g$

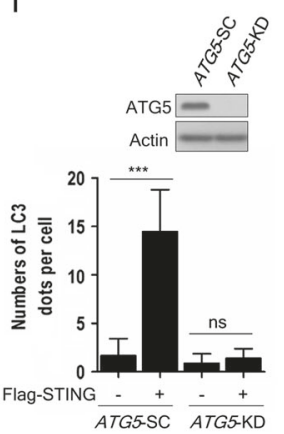

g
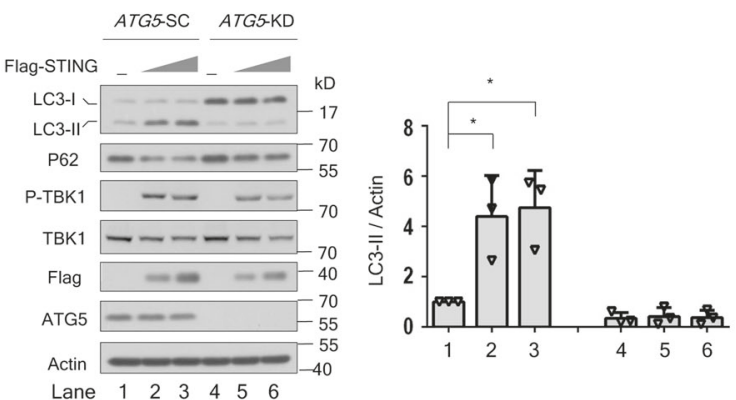

Fig. 2 STING overexpression induces ATG5-dependent autophagy. a HeLa and MEF cells were transfected with GFP-LC3 (green) plasmid together with an empty vector (Flag-3.0) or Flag-STING plasmid for $24 \mathrm{~h}$. The cells were fixed and stained with anti-Flag antibodies (red) and anti-p62 antibodies (purple), and images were then captured by confocal microscopy. Scale bar: $10 \mu \mathrm{m}$. b The numbers of GFPLC3 dots per cell in (a) were quantified (mean \pm s.d.; $n>100$ cells from three independent experiments). ${ }^{* * *} P<0.001$. c HeLa cells were transfected with a control Flag vector or a Flag-STING plasmid $(0.2$ $\mu \mathrm{g} / \mathrm{ml}$ and $0.5 \mu \mathrm{g} / \mathrm{ml}$, respectively) for $24 \mathrm{~h}$. Marker proteins for autophagy (LC3 and p62), mitochondria (ATPB) and the endoplasmic reticulum (Calnexin) were detected by immunoblotting analysis. Quantification of LC3-II expression levels in $\mathbf{c}$ is shown in the right panel (mean \pm s.d.; from three independent experiments). ${ }^{*} P<0.05$. d Immunoblot analysis of LC3-II expression in HeLa cells that were transfected with Flag vector or Flag-STING without or with

and 3a). As shown in Fig. 2e-g and Supplementary Fig. 3b, c STING-induced LC3 punctum formation and autophagic flux were blocked when ATG5 was knocked down in HeLa cells. Furthermore, poly(dA:dT) and cGAMP-induced autophagy were also blocked in $\operatorname{Atg} 5$ and $\operatorname{Atg} 7$ knockout MEF cells (Supplementary Fig. 3d-i). However, knockdown of BECN1 failed to prevent STING-induced and poly (dA:dT)-induced LC3-II conversion or the accumulation of LC3 dots (Fig. 3a-c, Supplementary Fig. 4a, and d). ULK1,

chloroquine (CQ) treatment at a final concentration of $25 \mu \mathrm{M} / \mathrm{ml}$. Quantification of LC3-II expression levels in $\mathbf{d}$ is shown in the right panel (mean \pm s.d.; from three independent experiments). ${ }^{*} P<0.05$. e HeLa cells expressing ATG5-SC (scramble control) and ATG5-KD shRNAs were co-transfected with GFP-LC3 plasmid, Flag vector or Flag-STING plasmid for $24 \mathrm{~h}$. The cells were fixed, stained and further visualized by confocal microscopy. Scale bar: $10 \mu \mathrm{m}$. f The numbers of GFP-LC3 dots per cell in e were quantified (mean \pm s.d.; $n>100$ cells from three independent experiments). ${ }^{* * *} P<0.001$. ns, not significant. The inset shows immunoblot analysis of ATG5 KD HeLa cells (expressing ATG5 shRNA) and control cells (expressing scramble shRNA). g Immunoblot analysis of LC3 protein levels in ATG5 KD and control ATG5 SC cells transfected with Flag-vector or Flag-tagged STING plasmid $(0.2 \mu \mathrm{g} / \mathrm{ml}$ and $0.5 \mu \mathrm{g} / \mathrm{ml}$, respectively). Quantification of LC3-II expression levels in $\mathbf{g}$ is shown in the right panel (mean $\pm \mathrm{s}$. d.; from three independent experiments). ${ }^{*} P<0.05$

the key kinase for autophagy initiation, was reported to phosphorylate STING and promote subsequent STING degradation to prevent sustained innate immune signaling [29]. However, knockout of ULK1 failed to block STINGinduced and poly(dA:dT)-induced LC3-II conversion and LC3 punctum formation, indicating that ULK1 is dispensable for STING-induced autophagy (Fig. 3d-f, Supplementary Fig. 4b, and e). Surprisingly, knockout of Atg9a also did not prevent STING-induced and poly(dA:dT)- 
a

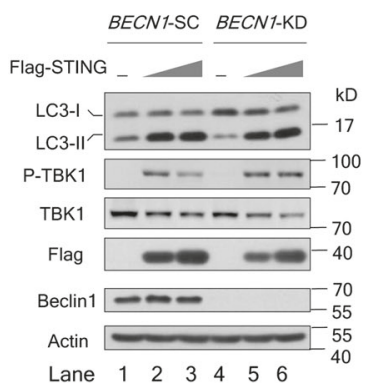

d
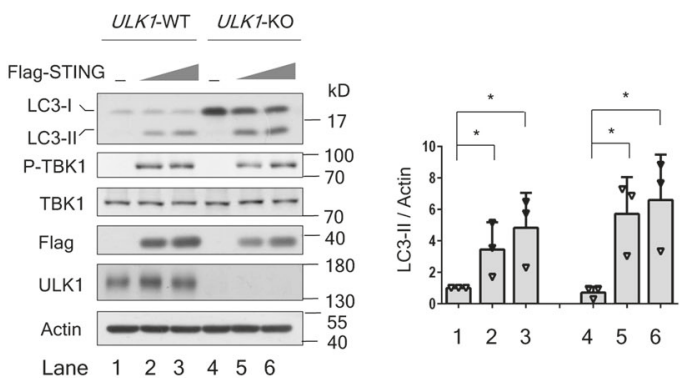

g

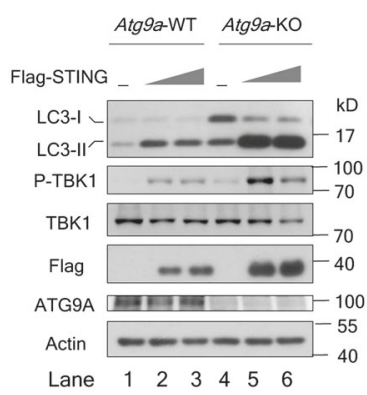

b

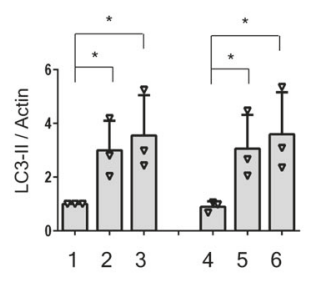

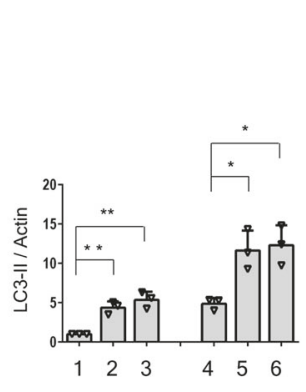

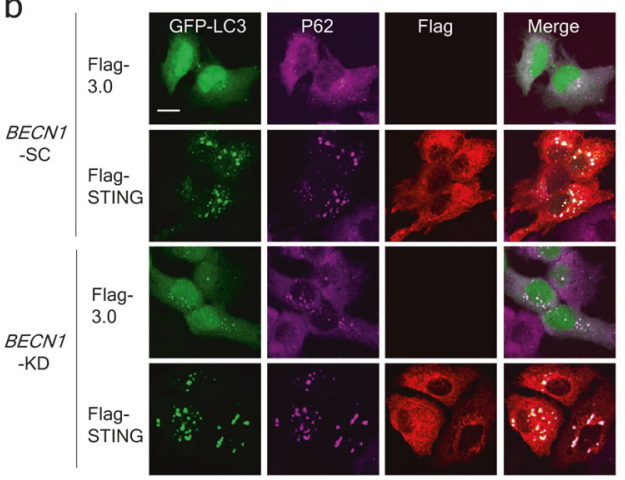

e

(Fla

ULK1
$-W T$

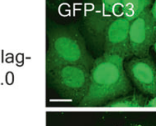

Flag-
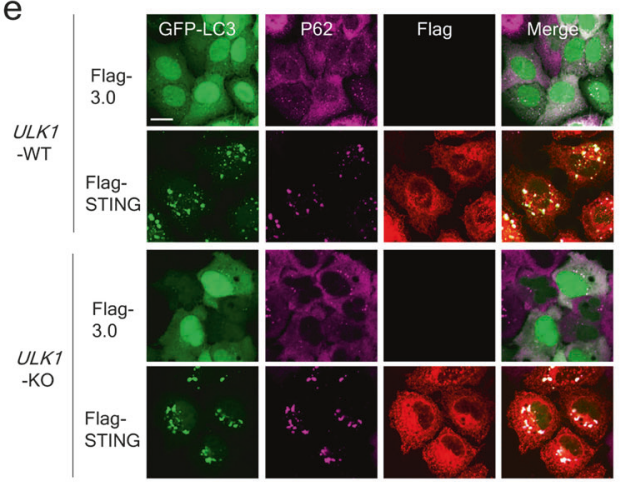

f

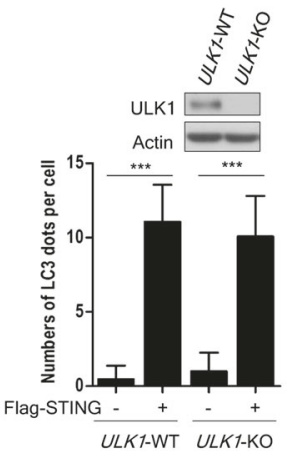

C

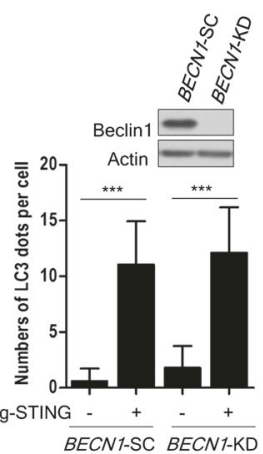

h

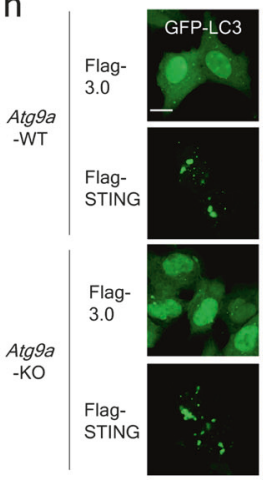

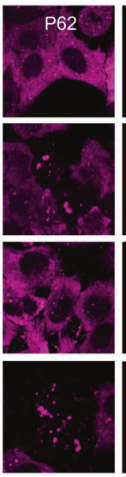

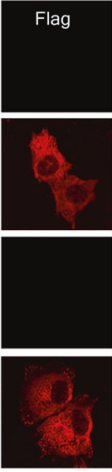

i

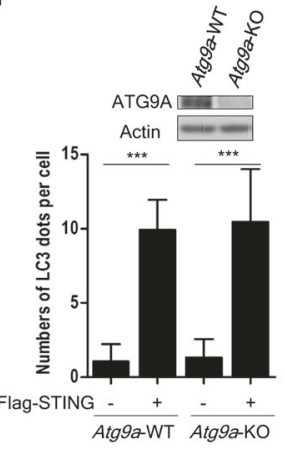

Fig. 3 STING-induced autophagy is independent of BECN1, ULK1 and Atg9a. a Immunoblot analysis of LC3-II expression levels in BECN1 knockdown cells and control cells transfected with Flag-vector or increasing amounts of Flag-STING plasmid. Quantification of LC3II expression levels in $\mathbf{a}$ is shown in the right panel (mean \pm s.d.; from three independent experiments). ${ }^{*} P<0.05$. b $B E C N 1$ knockdown cells and control cells were transfected with GFP-LC3 plasmid together with Flag-vector or Flag-STING plasmid. LC3 dot formation was examined by immunofluorescence microscopy. Scale bar: $10 \mu \mathrm{m}$. c The numbers of GFP-LC3 dots per cell in $\mathbf{b}$ were quantified (mean $\pm \mathrm{s}$. d.; $\mathrm{n}>100$ cells from three independent experiments). ${ }^{* * *} P<0.001$. The inset shows immunoblot analysis of BECN1 KD HeLa cells (expressing BECN1 shRNA) and control cells (expressing scramble shRNA). d Flag-vector and Flag-STING plasmids were transfected into $U L K 1$ wild-type and knockout cells for $24 \mathrm{~h}$, followed by western blotting assays of LC3-II conversion. Quantification of LC3-II expression levels in $\mathbf{d}$ is shown in the right panel (mean \pm s.d.; from three independent experiments). ${ }^{*} P<0.05$. e $U L K 1$ wild-type and knockout cells were transfected with GFP-LC3 and Flag-vector or Flag-STING plasmids, and images were captured by confocal microscopy. Scale bar: $10 \mu \mathrm{m}$. f Quantification of the numbers of LC3 dots per cell in e (mean \pm s.d.; $n>100$ cells from three independent experiments). ${ }^{* * *} P<0.001$. The inset shows immunoblot analysis of ULK1 knockout HeLa cells and wild-type cells. g Western blotting analysis of LC3-II expression levels in Atg9a wild-type and knockout HeLa cells transfected with Flag-vector or increasing amounts of FlagSTING plasmid. Quantification of LC3-II expression levels in $\mathbf{g}$ is shown in the right panel (mean \pm s.d.; from three independent experiments). ${ }^{*} P<0.05,{ }^{* *} P<0.01$. h Atg $9 a$ wild-type and knockout HeLa cells were transfected with plasmids expressing GFP-LC3 and Flag-vector or Flag-STING. Formation of LC3 puncta was detected by immunofluorescence microscopy. Scale bar: $10 \mu \mathrm{m}$. i Quantification of the numbers of LC 3 puncta per cell in $\mathbf{h}$ (mean \pm s.d.; $n>100$ cells from three independent experiments). ${ }^{* * *} P<0.001$. The inset shows immunoblot analysis of Atg $9 a$ knockout HeLa cells and wild-type cells 
induced autophagy (Fig. 3g-i, Supplementary Fig. 4c, and f), although a previous report suggested that Atg9a controls STING translocation from the Golgi apparatus and regulates the innate immune response [7]. P62 was reported to mediate STING degradation through autophagy [30]; however, we found that STING induced autophagy in $p 62$ knockout cells (Supplementary Fig. 4g-i). Collectively, these results show that STING induces a non-canonical form of autophagy that is dependent on ATG5, but not Beclin1, ULK1, Atg9a or p62.

\section{STING has autophagy receptor properties and directly interacts with LC3 via its LIR motifs}

Our results above revealed that STING plays a role in autophagy induction and is also subjected to autophagic degradation. One possibility is that STING functions as an autophagy receptor that directly interacts with LC3 to promote selective autophagy for subsequent autophagic degradation. We tested this hypothesis with coimmunoprecipitation experiments, which revealed that both endogenous LC3-II and GFP-tagged LC3 bind to ectopically expressed STING (Fig. 4a, b). Furthermore, pulldown analysis showed that LC3 and STING physically interact, as STING was pulled down by purified glutathione $S$-transferase (GST)-LC3, but not the GST control (Fig. 4c). It is well known that autophagy receptors such as p62 and Atg32 share a typical linear amino acid motif, called an LC3-interacting region (LIR), with a consensus sequence of W/YxxL/I [31, 32]. Using the iLIR server to predict potential LIR motifs in the STING protein [33], we identified seven putative LIRs (Fig. 4d and Supplementary Fig. 5a). To determine whether these LIR motifs are of functional importance for STING-induced autophagy, we generated a series of LIR mutations, named as mutLIR1-7, in which the first and the fourth conserved amino acids in the W/YxxV/L motif were mutated to alanine (A). Subsequently, these STING LIR mutants were transfected into Sting knockout HeLa cells. We found that the formation of LC3 puncta was greatly impaired in the presence of three single LIR mutants, mutLIR4, 6, and 7, and a triple-LIR mutant, mutLIR4/6/7 (Fig. 4e, f). These three LIR motifs are highly conserved from Danio rerio to Homo sapiens (Supplementary Fig. 5b). Biochemical analysis showed that mutLIR4/6/7 abolished the interaction between STING and LC3 and failed to induce the conversion of LC3 into LC3-II (Fig. 4g). Furthermore, purified GST-LC3 was able to pull down wild-type STING, but not the STING single LIR mutants LIR 4, 6, 7, or the triple LIR mutant LIR4/6/7 (Fig. 4h). Moreover, co-immunoprecipitation assays showed that more endogenous STING-LC3-II complex formed in response to poly $(\mathrm{dA}: \mathrm{dT})$ stimulation with chloroquine (CQ) treatment (Fig. 4i). To further demonstrate that STING acts as a specific autophagy receptor to mediate its own autophagic degradation, we performed rescue experiments in the Sting knockout cells. Wild-type STING restored the induction of LC3-II in poly(dA:dT)stimulated Sting knockout cells, whereas the mutLIR4/6/7 failed to do so (Fig. 4j). Importantly, wild-type STING, but not the mutLIR4/6/7 mutant, restored the induction of LC3II triggered by cGAMP in Sting knockout cells (Fig. 4k). Therefore, these data reveal that STING directly interacts with LC3 through its conserved LIR motifs to induce autophagy.

\section{STING activation is required for its autophagy induction}

It has been reported that STING-cGAMP complex formation and STING dimerization are essential for its activation and subsequent interferon production in response to cytosolic DNA [2, 5]. We observed that TBK1 activation occurred prior to autophagy induction following poly(dA: dT) and cGAMP treatments, which implies that STING activation is required for the induction of autophagy. To test this, we generated a series of mutations of the conserved residues that were reported to be critical for STING dimerization and cGAMP-binding [34-36]. These mutants greatly inhibited TBK1 phosphorylation and IFN- $\beta$ production (Fig. 5a, d and Supplementary Fig. 6). Firstly, we performed western blotting assays and found that overexpression of STING dimerization mutants, including the single site mutations (W161A, Y164A, and I165R) and the triple sites mutation (W161A/Y164A/I165R), failed to promote LC3-II conversion (Fig. 5a). Moreover, immunofluorescence microscopy showed that LC3 punctum formation was abolished in Sting knockout HeLa cells transfected with these dimerization mutants when compared with the wild-type STING (Fig. 5b). Accordingly, impaired STING dimerization disrupted STING-LC3 complex formation (Fig. 5c). In addition, cGAMPbinding mutants of STING, including the single site mutations (S162Y, Y167W, Y240S, and E260A) and the tetrad sites mutation (S162Y/Y167W/Y240S/E260A), were transfected into Sting knockout HeLa cells, which were further subjected to immunoblotting analysis. Compared to wild-type STING, these mutants abolished LC3-II conversion, suggesting that cGAMP-STING interaction is required for autophagy induction (Fig. 5d). In addition, these mutants significantly reduced the accumulation of LC3 dots and attenuated the formation of STING-LC3 complexes (Fig. 5e, f). Taken together, these results indicate that STING dimerization and cGAMP binding are required for autophagy induction. 
a

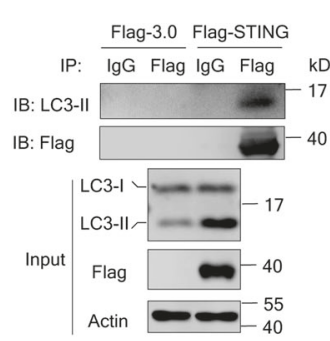

d

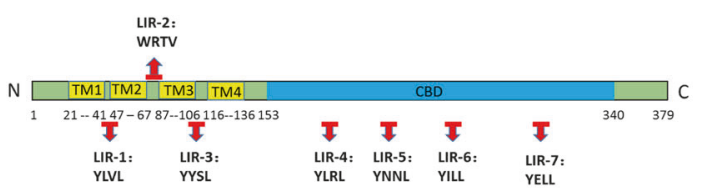

g
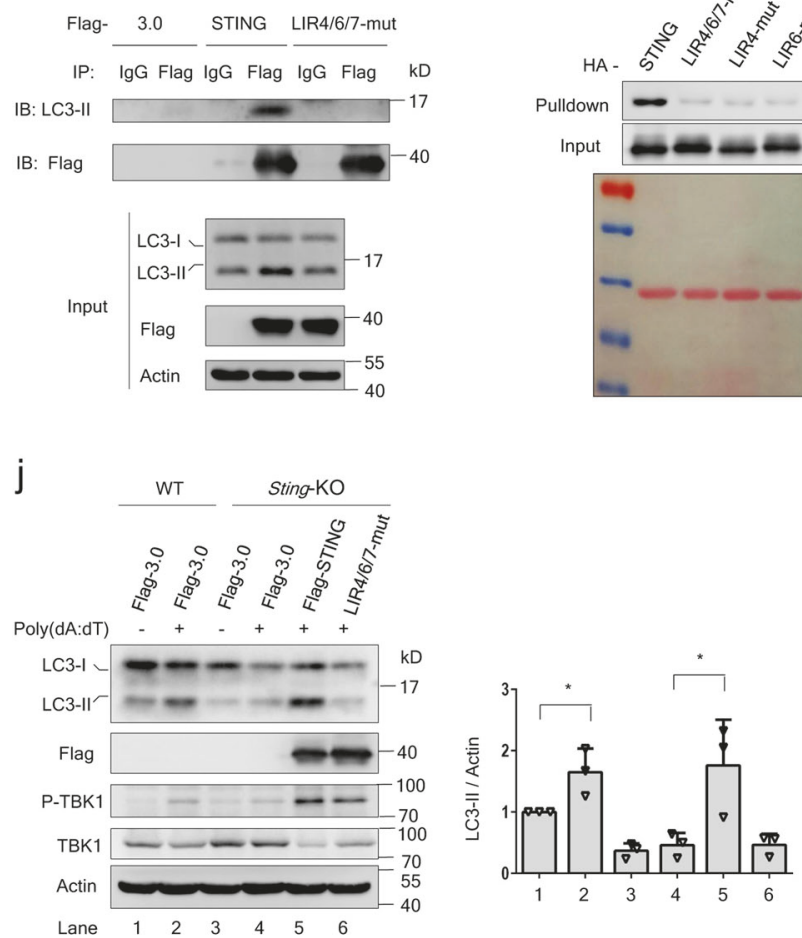

\section{TBK1 and IRF3 are not required for STING-induced autophagy}

As TBK1 is able to phosphorylate p62 and OPTN [24, 25], two important autophagy receptors, we next asked whether STING downstream signaling is involved in STINGinduced autophagy. RNA interference (RNAi) experiments to knock down the STING downstream kinase TBK1 and the transcription factor IRF3 had no obvious effects on STING-induced autophagy (Fig. 6a, b). Neither TBK1 nor

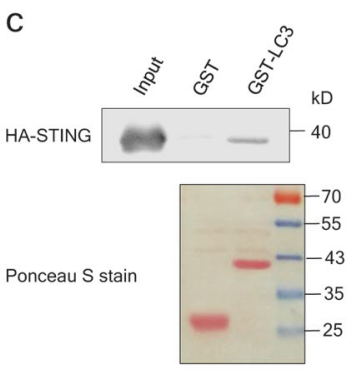

e
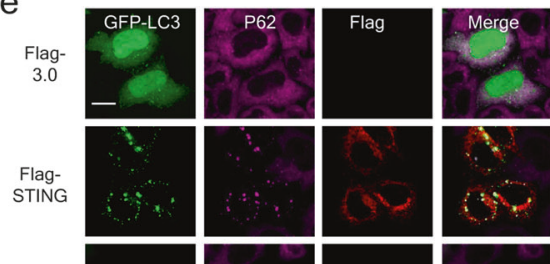

LIR4-
mut
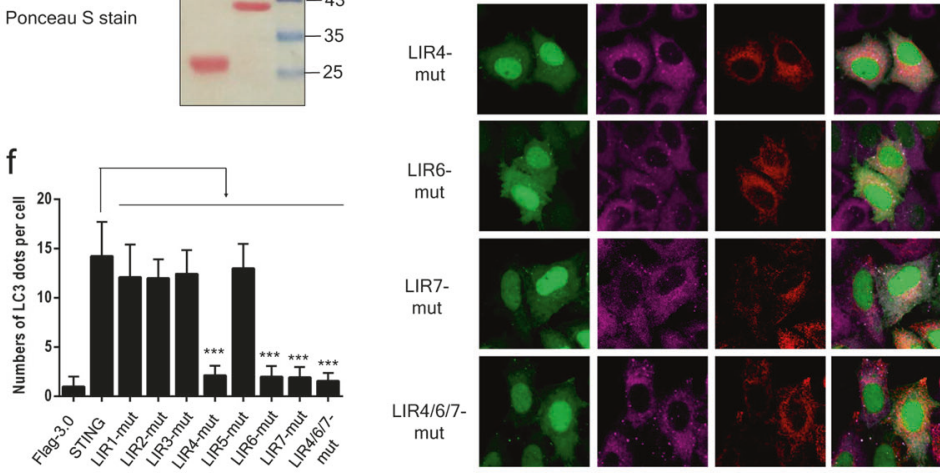

LIR6-
mut
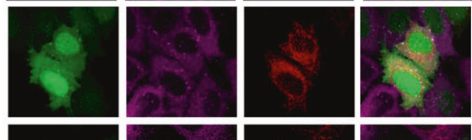

LIR7-
mut
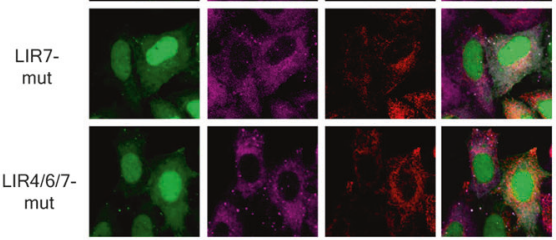

i
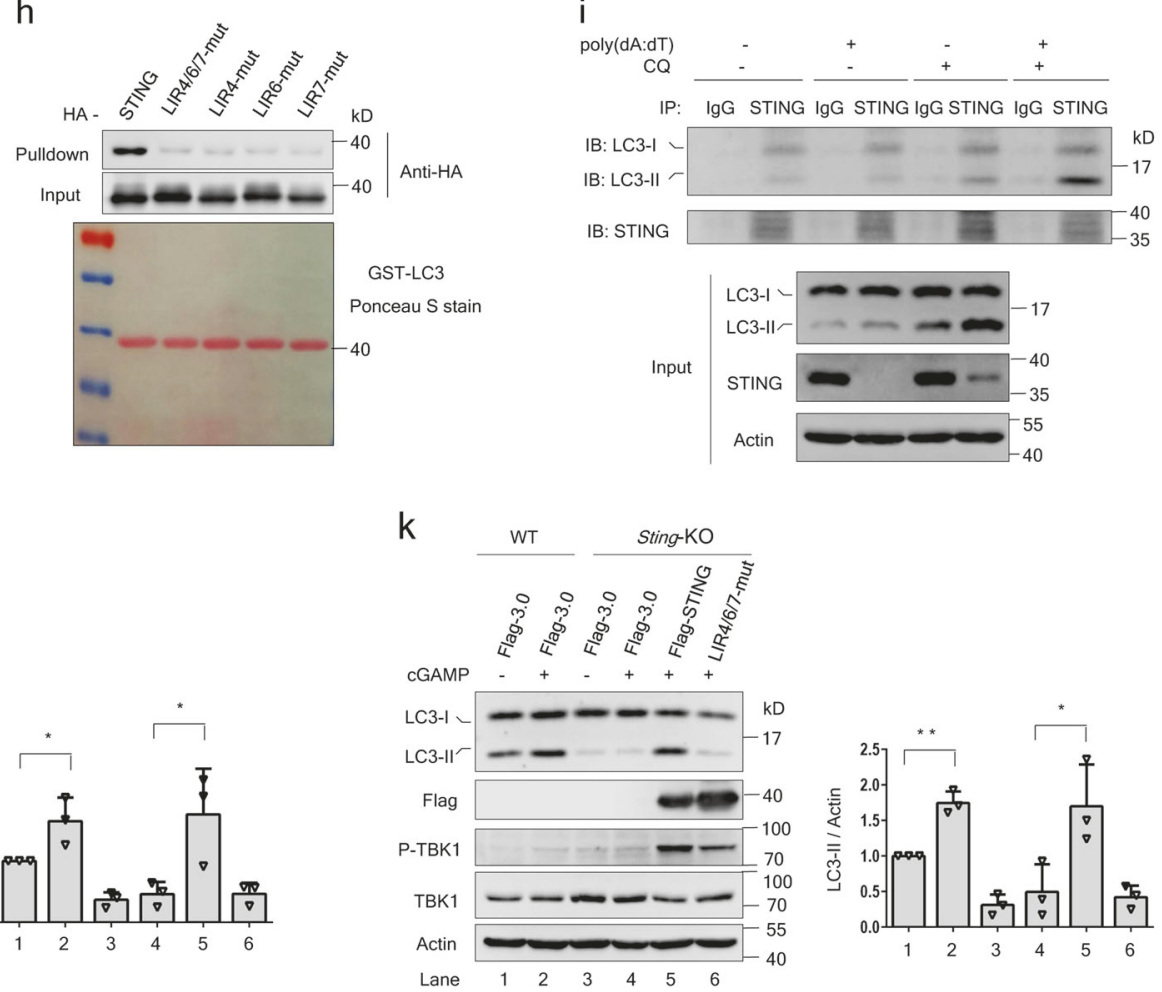

IRF3 deficiency blocked the increase of LC3-II levels and LC3 punctum formation induced by poly(dA:dT) stimulation (Fig. 6c-f). It has been reported that the C-terminus of STING (aa341-379) is required and sufficient for interactions with TBK1 and IRF3, which facilitate IRF3 activation by TBK1 [13]. As shown in Fig. 6g, the C-terminal truncation $(\Delta 340-379)$ of STING was unable to activate downstream phosphorylation of TBK1 and IRF3. Similar to wild-type STING, this truncation still effectively promoted LC3-II conversion and LC3 punctum formation (Fig. 6g, h). 
Fig. 4 STING has autophagy receptor properties and directly interacts with LC3 via its LIR motifs. a Sting knockout HeLa cells were transfected with the indicated plasmids for $24 \mathrm{~h}$. Cells lysates were incubated with protein $\mathrm{G}$ agarose beads and indicated antibody at $4{ }^{\circ} \mathrm{C}$ overnight, then immunoblotted. b Sting knockout HeLa cells were cotransfected with GFP-LC3 and Flag-vector or Flag-STING plasmids. Cell lysates were subjected to immunoprecipitation with protein $G$ agarose beads and immunoblot analysis with anti-GFP. c Purified GST or GST-LC3 proteins were incubated with lysates of HeLa cells transfected with a plasmid expressing HA-STING at $4{ }^{\circ} \mathrm{C}$ overnight. Immunoblot analysis was then carried out to detect the interaction between STING and LC3. Ponceau S staining shows the abundance of the GST and GST-LC3 proteins. d Graphical representation of the STING protein showing the three domains and the seven potential LIR motifs. The N-terminal domain contains 4 transmembrane regions (TM1-4), the CBD domain binds to c-di-GMP, and the C-terminal domain contains the cytoplasmic tail. The amino acid sequences of the predicted LIR motifs are indicated. e Sting knockout HeLa cells were transfected with plasmids encoding GFP-LC3 and wild-type STING or its LIR motif mutants. Formation of LC3 puncta was detected by immunofluorescence microscopy. f Quantification of the numbers of LC3 dots per cell (mean \pm s.d.; $n>100$ cells from three independent experiments). Scale bar: $10 \mu \mathrm{m}$. ${ }^{* * *} P<0.001$. g Sting knockout HeLa cells were transfected with control vector (Flag-3.0), or plasmids expressing wild-type Flag-STING or its triple LIR motif mutant. Cell lysates were subjected to immunoprecipitation and immunoblot analysis of the interaction between LC3 and STING or its mutants. h GST-fused LC3 proteins were incubated with lysates of HeLa cells transfected with plasmids carrying genes for wild-type STING or the indicated mutants at $4{ }^{\circ} \mathrm{C}$ overnight. Immunoblotting was then performed to analyze the association between LC3 and STING or its mutants. Ponceau S staining shows the abundance of GST-LC3 proteins. i MEF cells were stimulated with or without poly(dA:dT) or chloroquine (CQ), then lysates were subjected to coimmunoprecipitation and immunoblotting to analyze the interaction between endogenous STING and LC3. j Immunoblot analysis of LC3II expression levels in Sting wild-type and knockout HeLa cells expressing the indicated plasmids with or without poly(dA:dT) stimulation. Quantification of LC3-II expression levels in $\mathbf{j}$ is shown in the right panel (mean \pm s.d.; from three independent experiments). ${ }^{*} P$ $<0.05$. k Immunoblot analysis of LC3-II conversion in Sting wild-type and knockout HeLa cells transfected with the indicated plasmids with or without cGAMP stimulation. Quantification of LC3-II expression levels in $\mathbf{k}$ is shown in the right panel (mean \pm s.d.; from three independent experiments). ${ }^{*} P<0.05,{ }^{* *} P<0.01$

Accordingly, co-immunoprecipitation experiments showed that the truncated STING interacted with LC3 (Fig. 6i). Collectively, our findings suggest that TBK1 and IRF3 are dispensable for STING-induced autophagy.

\section{HSV-1 induces STING-dependent autophagy}

It is well-established that STING is triggered by a variety of DNA viruses, such as Herpes Simplex Virus (HSV-1). We next sought to demonstrate that STING induces autophagy upon HSV-1 infection. Western blot assays showed that HSV-1 induced LC3-II conversion in wild-type cells, but not in Sting knockout cells (Fig. 7a). In addition, HSV-1 infection significantly increased LC3 punctum formation and autophagic flux in wild-type but not Sting knockout cells (Fig. 7b-d). Inhibition of autophagy by chloroquine led to the restoration of STING expression in HSV-1infected MEF cells (Fig. 7d). In order to further verify that HSV-1-induced autophagy depends on STING, we carried out rescue experiments in Sting knockout cells. The failure of HSV-1 infection to induce LC3-II conversion in Sting knockout cells was corrected by introduction of wild-type STING but not the triple LIR mutant (Fig. 7e). Collectively, these data showed that STING induces autophagy during the process of viral infection.

\section{Discussion}

In the present study, we provide evidence to show that STING functions as a potential autophagy receptor and directly interacts with LC3 to mediate autophagy and its own autophagic degradation in a non-canonical fashion. We observed that the STING activators poly(dA:dT), cGAMP and HSV-1 induced STING-dependent autophagy. Also, STING deficiency failed to block starvation-induced autophagy, suggesting that STING is specifically essential for immunogenic stimulation-induced autophagy (Supplementary Fig. 7). We identified that STING contains conserved LIR motifs that directly interact with LC3 for the activation of autophagy, leading to the degradation of STING itself and p-TBK1 (Supplementary Fig. 1 and 2f, Fig. 1). Mutation of STING LIR motifs abolished its interaction with LC3 and its capacity to restore autophagy induction in Sting knockout cells. We also found that HSV1 infection induces STING-dependent autophagy, and wild-type STING, but not its LIR mutants, restored the induction of autophagy in Sting knockout cells during HSV-1 infection. Interestingly, we found that ectopic expression of STING induces autophagy even in the absence of p62 (Supplementary Fig. 4g-i). It is possible that p62 is necessary, but not sufficient, for STINGdependent autophagy. Recent study by Thaneas Prabakaran et al. shows that STING is targeted for autophagic degradation in a p62-dependent manner [30]. They showed that in response to dsDNA treatment STING is ubiquitinated and recruited to p62-positive compartments. STING fails to tracffic to autolysosome in p62-deficient cells, suggesting that p62 facilitates STING degradation via autophagosome formation. We did observe that p62 colocalizes with STING and becomes degraded when STING is activated (Fig. 2). It appears that the ubiquitination of STING helps the recruitment of $\mathrm{p} 62$, a well characterized autophagy receptor, to facilitate autophagic degradation of STING for fine tuning the innate immune response. More studies are warranted to understand the interplay between STING and p62 for their involvements in autophagy and immune regulation. It is also surprising to find that STING activates 

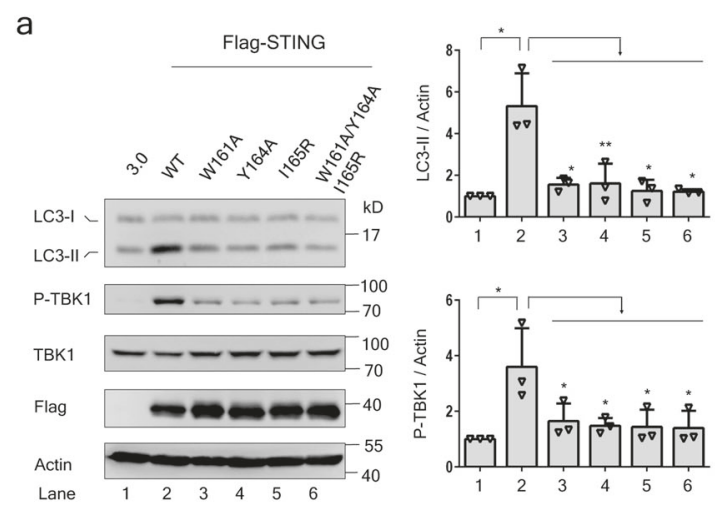

b

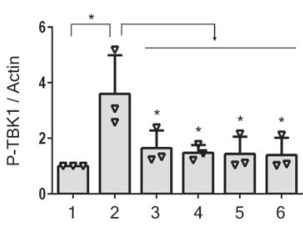

C

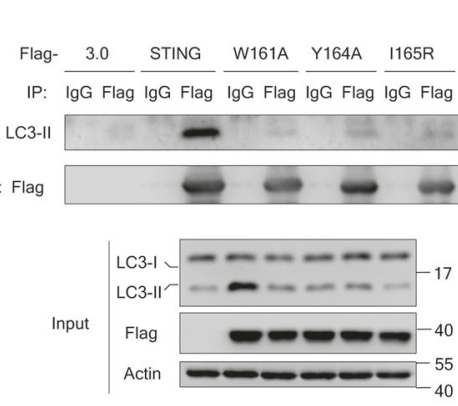

d
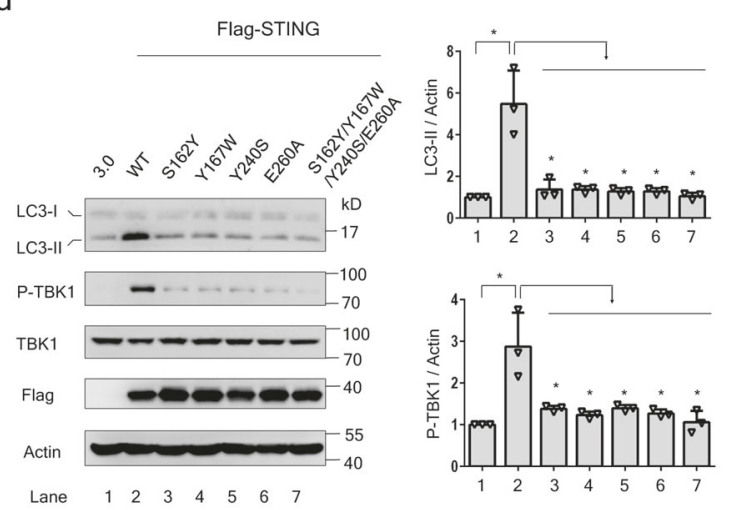

f

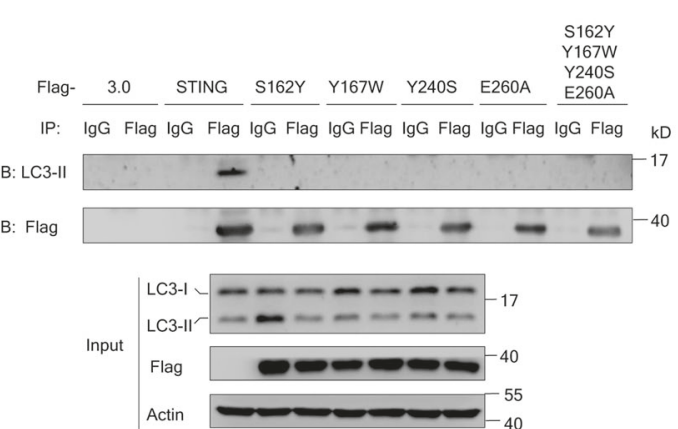

Flag-3.0

Flag-STING
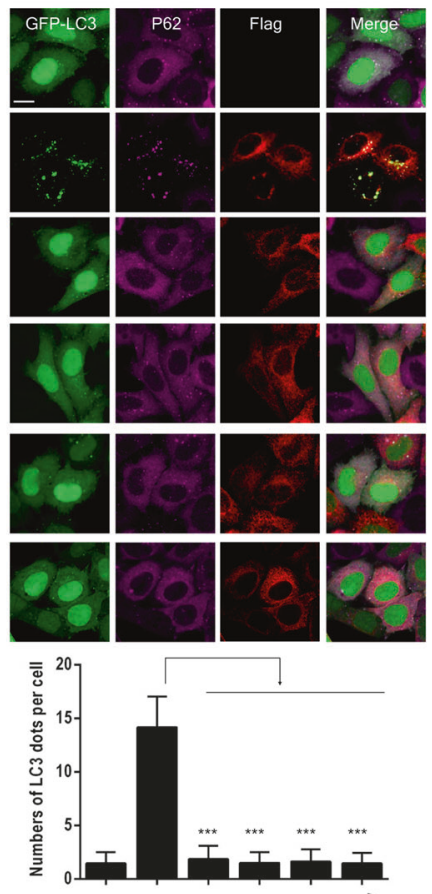

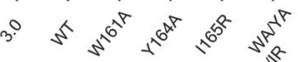

e

Flag-30
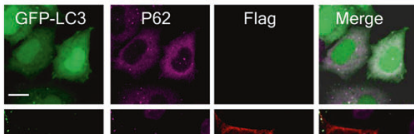

Flag-STING
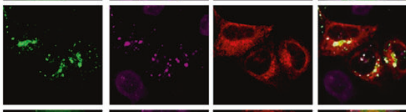

Flag-STING
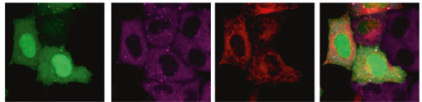

Flag-STING
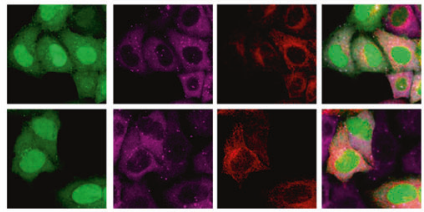

Flag-STING

Flag-STING

$-E 260 \mathrm{~A}$
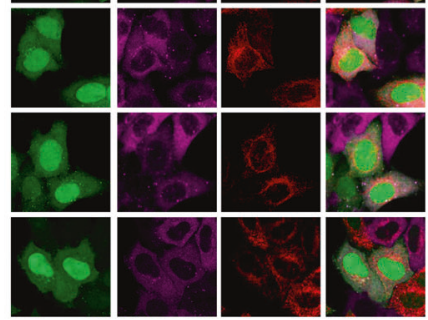
240S/E260A

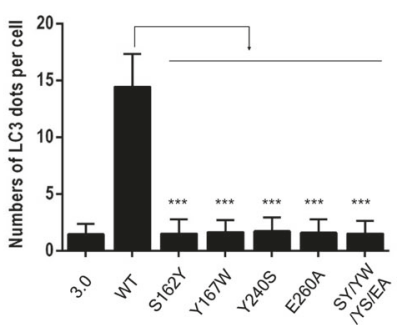

autophagy in the absence of ULK1, Beclin1, and Atg9a (Fig. 3). Previous reports have shown that ULK1 is able to phosphorylate STING on S366 to promote subsequent
STING degradation, and Atg9a controls translocation of STING [7, 29]. We found that the S366A mutant of STING is still able to effectively promote LC3-II conversion and 
Fig. 5 STING activation is essential for the activation of autophagy. a Sting knockout HeLa cells were transfected with plasmids encoding wild-type Flag-STING or its dimerization mutants (W161A, Y164A, I165R, and W161A/Y164A/I165R) for $24 \mathrm{~h}$. Lysates were then immunoblotted to analyze LC3-II conversion. Quantification of LC3-II and P-TBK1 expression levels in $\mathbf{a}$ is shown in the right panel (mean \pm s.d.; from three independent experiments). ${ }^{*} P<0.05,{ }^{* *} P<0.01$. b Sting knockout HeLa cells were transfected with plasmids expressing GFP-LC3 together with control vector (Flag-3.0), wild-type FlagSTING or the indicated STING dimerization mutants. LC3 punctum formation was assessed by immunofluorescence microscopy. Scale bar: $10 \mu \mathrm{m}$. Quantification of the numbers of LC3 puncta per cell in $\mathbf{b}$ is shown below the confocal images (mean \pm s.d.; $n>100$ cells from three independent experiments). ${ }^{* * *} P<0.001$. c Sting knockout HeLa cells were transfected with the indicated plasmids for $24 \mathrm{~h}$, and the cell lysates were incubated with protein $\mathrm{G}$ agarose beads and indicated antibody at $4{ }^{\circ} \mathrm{C}$ overnight, then immunoblotted to analyze the interaction between LC3 and STING or its dimerization mutants. d Immunoblot analysis of the expression levels of LC3-II in Sting knockout HeLa cells transfected with Flag-tagged STING or its cGAMP-binding mutants (S162Y, Y167W, Y240S, E260A, and S162Y/Y167W/Y240S/E260A). Quantification of LC3-II and P-TBK1 expression levels in $\mathbf{d}$ is shown in the right panel (mean \pm s.d.; from three independent experiments). ${ }^{*} P<0.05$. e Plasmids encoding GFPLC3 together with wild-type STING or its cGAMP-binding mutants were transfected into Sting knockout HeLa cells for $24 \mathrm{~h}$, and the LC3 dots were detected by immunofluorescence microscopy. Scale bar: 10 $\mu \mathrm{m}$. Quantification of the numbers of LC3 dots per cell in $\mathbf{e}$ is shown below the confocal images (mean \pm s.d.; $n>100$ cells from three independent experiments). ${ }^{* * *} P<0.001$. f Co-immunoprecipitation and immunoblot analysis of the interaction between LC3 and STING or its cGAMP-binding mutants in Sting knockout HeLa cells

LC3 punctum formation (data are not shown), suggesting that ULK1-mediated STING phosphorylation on S366 is dispensable for STING-induced autophagy. Tatsuya Saitoh et al. reported that Atg9a limits dsDNA-induced innate immune responses by regulating the assembly of STING and TBK1 without affecting the translocation of STING from the ER to the Golgi apparatus [7]. However, the precise mechanism by which Atg9a affects STING is not clear. We and others have shown that Atg9a traffics between the plasma membrane and TGN to deliver membranes as a source for autophagosome formation [37-39]. Several other selective auto(mito-)phagy receptors, such as FUNDC1, mediate auto(mito-)phagy in the absence of BECN1 gene [40]. A recent report shows that STING mediates a constitutive cell-autonomous phagocyte response specifically to Gram-positive bacteria and functions to alleviate ER stress by removing stressed ER membranes (ER-phagy) [28]. STING exhibits a punctate expression pattern reminiscent of autophagosomes, and colocalizes with several autophagy proteins such as p62 and LC3 upon activation (Tatsuya Saitoh et al., 2009, and our study) [7]. As the ER is a major membrane source for autophagosome formation, it is possible that STING activates the ATG5-dependent core autophagy machinery to regulate innate immune signaling.
Our results uncover dual roles of STING in immune activation and autophagy. We observed that STING activation is an early event, which is immediately followed by its own autophagic degradation together with degradation of $\mathrm{p}$-TBK1. Under physiological conditions, STING exists as a weak dimer. Upon binding of activators or ligands, STING undergoes activation and translocation from the ER to the Golgi apparatus and the perinuclear compartments, which further activates the TBK1-IRF3 axis, resulting in type I IFN production [10]. Extensive biochemical and structural studies have identified some critical dimerization and c-di-GMP binding sites of STING [34-36]. We showed that a series of mutations that abolish STING dimerization and cGAMP-binding also failed to induce LC3-II conversion and LC3 punctum formation, and prevented STING from interacting with LC3. This suggests that the cGAMP binding and dimerizing activities are indispensable for STING-induced autophagy. Further structural analysis revealed that the LIR motifs of STING are partially exposed to the cytosol upon activation (data are not shown), providing an effective platform for the direct interaction of STING with LC3. We also noted that phosphorylated TBK1 is subjected to STING-induced autophagic degradation, whereas the unphosphorylated form remains stable (Fig. 1g, h, and Supplementary Fig. 2f). We found that inhibition of autophagy by chloroquine significantly reduced the degradation of STING and p-TBK1 (Fig. 1g, h), while the proteasome inhibitor MG132 had limited effect. This implies that only the activated TBK1 within the STING-p62 complex is prone to autophagic degradation. It is possible that STING-TBK1 interaction may facilitate the exposure of the LIR motifs in STING. Such a mechanism is consistent with literature showing that autophagy, namely the ATG gene products such as ATG5 and ATG16L1, plays an active role in preventing unnecessarily over-exuberant inflammatory responses and resistance to pathogens infection to ensure a measured response [19, 41-43]. Our results further extended these findings by suggesting that STING directly couples immune activation with autophagy to negatively regulate the immune response. Further studies will be directed towards understanding the structural basis of the STINGTBK1 interaction for immune activation and the STINGLC3 interaction for autophagy induction.

\section{Materials and methods}

\section{Antibodies and reagents}

The rabbit antibodies against TBK1 (3013), IRF3 (4302), pIRF3 (Ser396, 4947), STING (13647), and ATG5 (12994) 

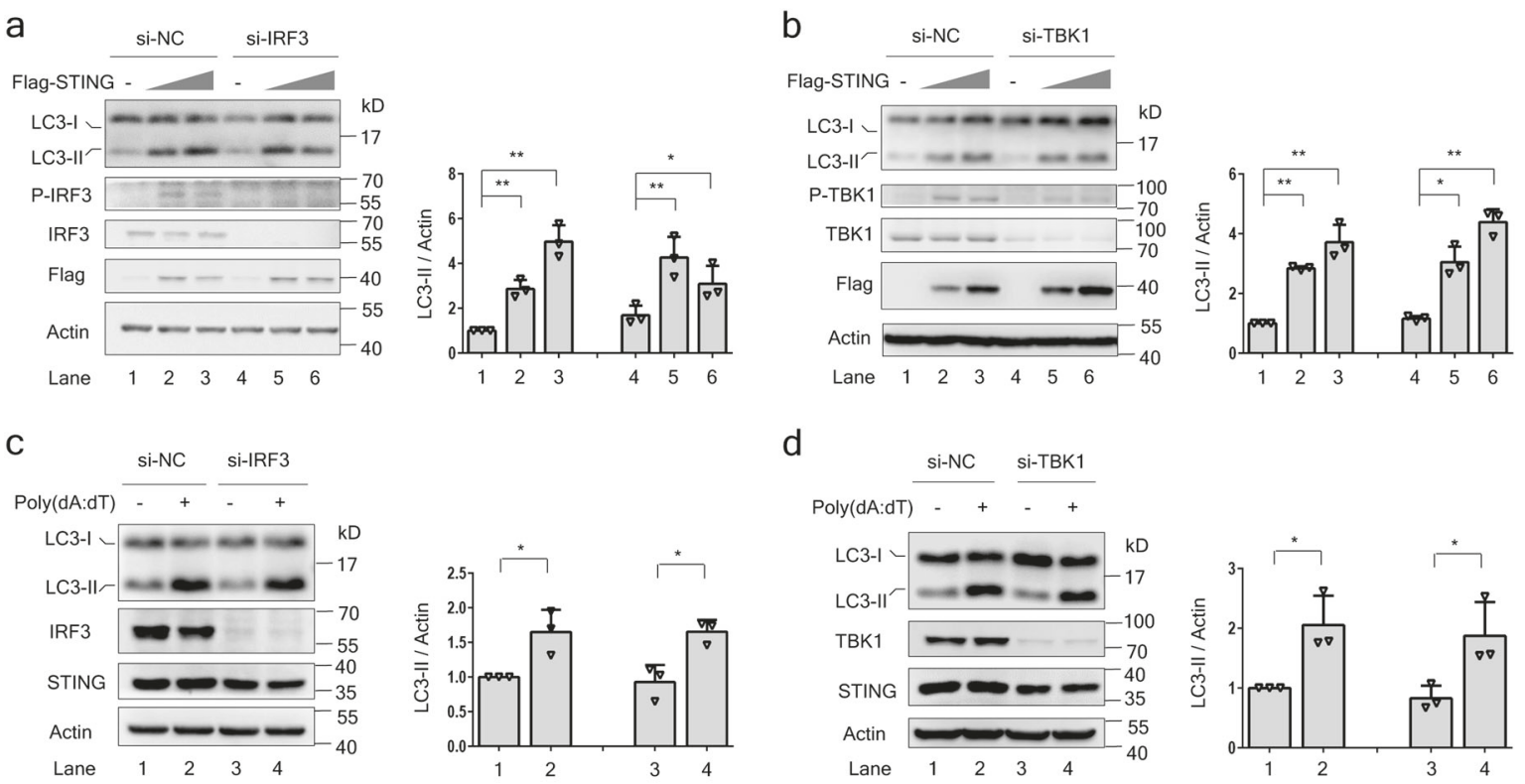

e
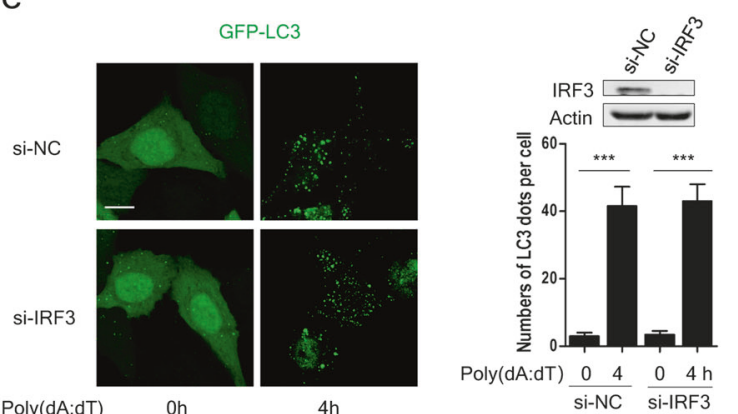

f
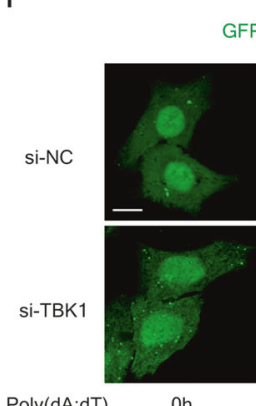

GFP-LC3
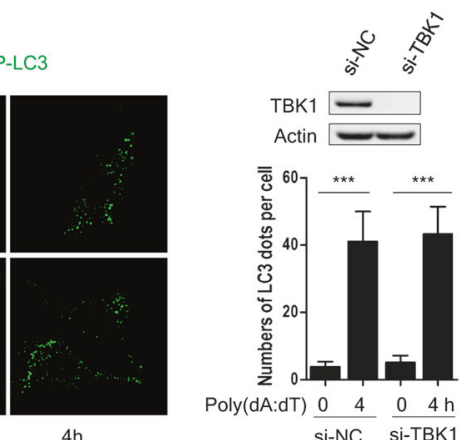

g

$\mathrm{h}$
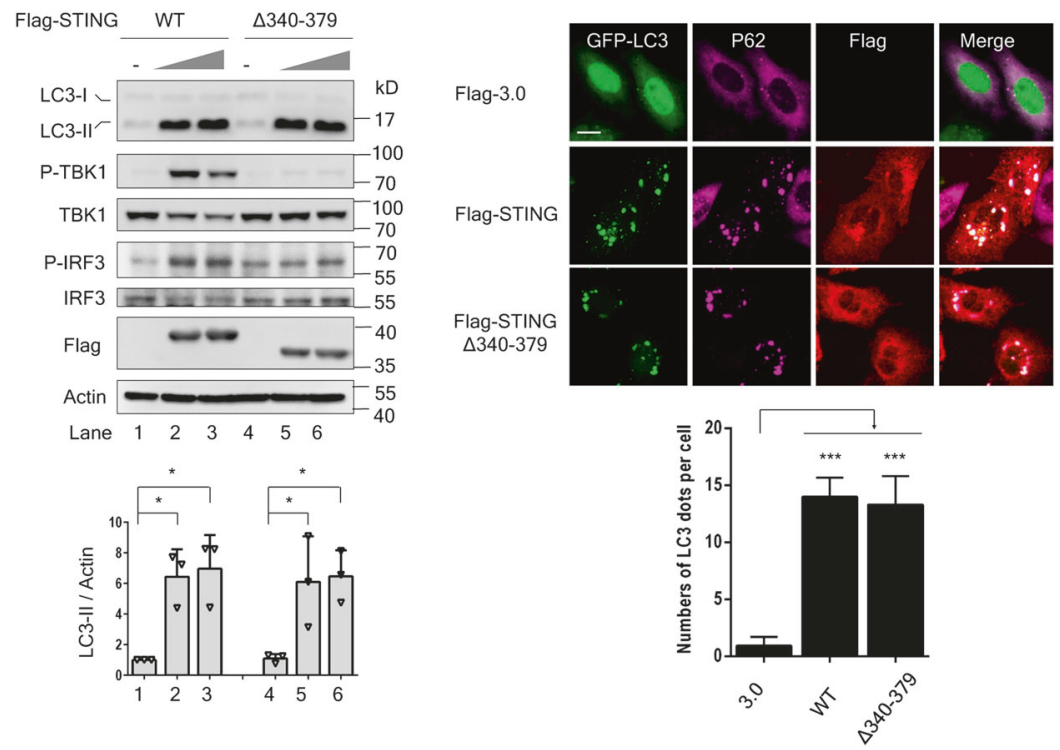

i
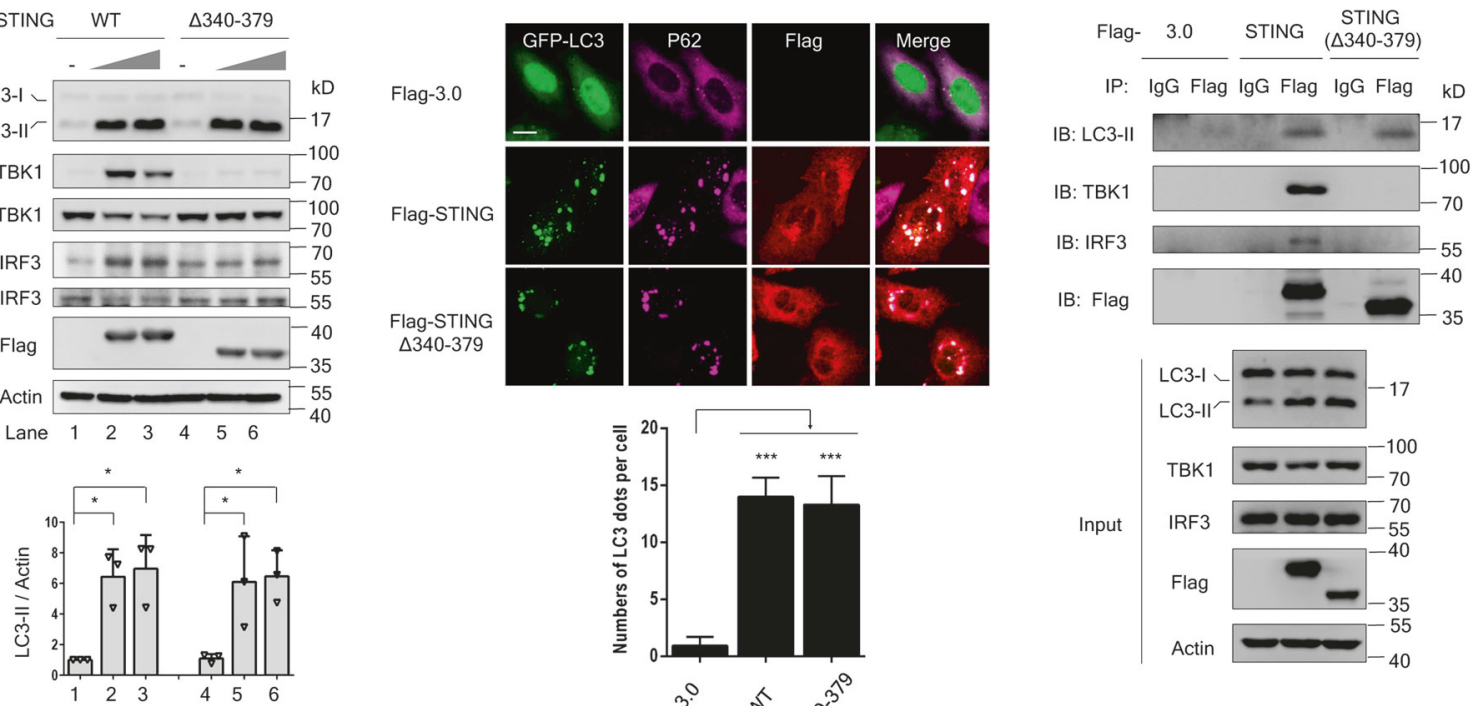

were purchased from Cell Signaling Technology. The mouse anti-GFP (sc-9996), the rabbit anti-ULK1 (sc33182), anti-rabbit IgG (sc-2027), anti-mouse IgG (sc-
2025), and protein $G$ agarose beads were obtained from Santa Cruz. The mouse antibodies specific for Beclin-1 (612113) and calnexin (610523) were from BD 
Fig. 6 TBK1 and IRF3 are not required for STING-induced autophagy. a-b Immunoblot analysis of LC3-II conversion in HeLa cells transfected with the indicated siRNAs and increasing amounts of STING plasmid. Quantification of LC3-II expression levels in $\mathbf{a}-\mathbf{b}$ is shown in the right panel (mean \pm s.d.; from three independent experiments). ${ }^{*} P<0.05,{ }^{* *} P<0.01$. c-d HeLa cells were transfected with the indicated siRNAs, then stimulated with poly(dA:dT) or not. The LC3-II expression levels were evaluated by western blotting. Quantification of LC3-II expression levels in c-d is shown in the right panel (mean \pm s.d.; from three independent experiments). ${ }^{*} P<0.05$. e-f HeLa cells stably expressing GFP-LC3 were transfected with the indicated siRNAs and subsequently stimulated with poly(dA:dT) for 4 h. LC3 dot formation was observed by immunofluorescence microscopy. Quantification of the numbers of LC3 puncta per cell and the knockdown efficiency of IRF3 and TBK1 are shown on the right (mean \pm s.d.; $n>100$ from three independent experiments cells). Scale bar: $10 \mu \mathrm{m} .{ }^{* * *} P<0.001$. g HeLa cells were transfected with increasing amounts of plasmids expressing wild-type Flag-STING or the Cterminal truncation $(\Delta 340-379)$. Cell lysates were subjected to western blotting analysis with the indicated antibodies. Quantification of LC3II expression levels in $\mathbf{g}$ is shown below the Fig. $6 \mathrm{~g}$ (mean \pm s.d.; from three independent experiments). ${ }^{*} P<0.05$. h HeLa cells were cotransfected with plasmids encoding GFP-LC3 and wild-type FlagSTING or the C-terminal truncation mutant. Formation of LC3 puncta was then observed by immunofluorescence microscopy. Scale bar: 10 $\mu \mathrm{m}$. Quantification of the numbers of LC3 dots per cell in $\mathbf{h}$ is shown below the confocal images (mean \pm s.d.; $n>100$ cells from three independent experiments). ${ }^{* * *} P<0.001$. i Co-immunoprecipitation and immunoblot analysis of the interaction between LC3 and STING or its C-terminal truncation $(\Delta 340-379)$ in Sting knockout HeLa cells

Biosciences. The following antibodies were purchased from Sigma: anti-LC3 (L8918), anti-Flag (F1804), anti-Actin (A8481), and anti-HA (H9658). The rabbit anti-ATG9A (ab108338) and anti-p-TBK1 (Ser172, ab109272) were bought from Abcam. The rabbit antibody against ATPB (17247-1-AP) and ATG7 (10088-2-AP) was from Proteintech, and the rabbit anti-p62 (PM045) was purchased from MBL. The protein A agarose beads were bought from Invitrogen. The secondary antibodies used for immunofluorescence in this study were as follows: goat anti-mouse IgG Alexa Fluor-568 (Molecular Probes, A11004) and antirabbit IgG Alexa Fluor-647 (Molecular Probes, A21244). Earle's Balanced Salt Solution (EBSS) (SH30014.08) was from HyClone. Dual-luciferase reporter assay system was ordered from Promega.

Both poly(dA:dT) (tlrl-patn-1) and cGAMP (tlrlnacga23-1) were purchased from InvivoGen, and were used at a final concentration of $1 \mu \mathrm{g} / \mathrm{ml}$ or $5 \mu \mathrm{g} / \mathrm{ml}$ for MEF cells or Hela cells, respectively. Chloroquine and MG132 were bought from Sigma and used at a final concentration of 20 $\mu \mathrm{M} / \mathrm{ml}$ or $10 \mu \mathrm{M} / \mathrm{ml}$.

\section{Plasmids and virus}

Flag-STING, IFN- $\beta$ luciferase reporter gene and pRL-TK plasmids were kindly provided by Dr Shuai Chen (Sun Yatsen University Cancer Center, Guangzhou, China). HASTING plasmid was a gift from Dr Zhengfan Jiang (Peking
University, Beijing, China). STING mutants (LIR1-7 mutants, LIR4/6/7 mutant, W161A, Y164A, I165R, W161A/Y164A/I165R, S162Y, Y167W, Y240S, E260A, $\mathrm{S} 162 \mathrm{Y} / \mathrm{Y} 167 \mathrm{~W} / \mathrm{Y} 240 \mathrm{~S} / \mathrm{E} 260 \mathrm{~A}$, and $\Delta 340-379)$ were generated according to the manufacturer's (Stratagene, 200521) protocols. HSV-1 virus was kindly provided by Dr Hongyu Deng (Institute of Biophysics, Chinese Academy of Sciences, Beijing, China).

\section{Cell culture and transfection}

Sting wild-type and knockout MEF and HeLa cells were shared by Dr Zhengfan Jiang (Peking University, Beijing, China) [44]. The following HeLa cell lines were generated in the lab: p62-knockout, ULK1-knockout, Atg9a-knockout, ATG5-knockdown, and BECN1-knockdown. MEF cells, HeLa cells, MCF-7 cells, and HepG2 cells were cultured in Dulbecco's modified Eagle's medium (DMEM, Invitrogen) supplemented with $10 \%$ fetal bovine serum (FBS, Hyclone) at $37{ }^{\circ} \mathrm{C}$ in $5 \% \mathrm{CO}_{2}$. Indicated plasmids were transfected into cells with MegaTran 1.0 (OriGene) according to the manufacturer's instructions. Poly(dA:dT) and cGAMP were transfected into cells using Lipofectamine 2000 according to the manufacturer's protocols.

\section{Immunoprecipitation and immunoblotting}

Cells were harvested and lysed with lysis buffer $(20 \mathrm{mM}$ Tris, pH7.4, 2 mM EGTA, 1\% NP-40) plus protease inhibitors. After centrifugation, the lysates were incubated with specific antibodies and protein A beads (Invitrogen) or protein $\mathrm{G}$ beads (Santa Cruz) at $4{ }^{\circ} \mathrm{C}$ overnight. The beads were washed three times with lysis buffer, followed by immunoblotting analysis with the indicated antibodies. For the immunoblotting, cells were collected and lysed with lysis buffer, and then lysates were run on SDS-PAGE and transferred to nitrocellulose membranes. The membranes were blocked with 5\% fat-free milk for $2 \mathrm{~h}$ at room temperature, then incubated with the indicated primary antibodies at $4{ }^{\circ} \mathrm{C}$ overnight and HRP-conjugated secondary antibodies for $3 \mathrm{~h}$ at room temperature. Immunoreactive bands were visualized with a chemiluminescense kit (Tanon, Shanghai, China).

\section{RNA interference (RNAi)}

The siRNAs used in this study were all synthesized by RiboBio (Guangzhou, China). Cells were transfected with RNAi oligonucleotides using Lipofectamine 2000 reagent (Invitrogen) according to the manufacturer's instructions. The RNAi sequences used in this study were as follows: siTBK1, 5'- CACAAAUUUGAUAAGCAAA-3'; and siIRF3, 5'-GGAGUGAUGAGCUACGUGA-3'. 
a

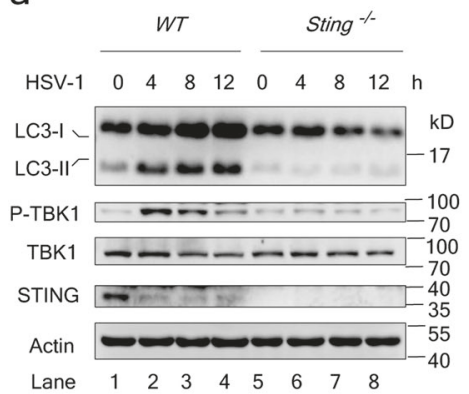

d

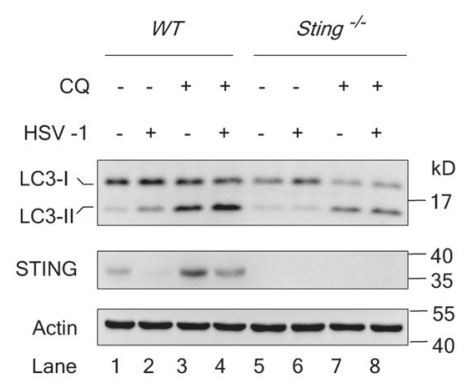

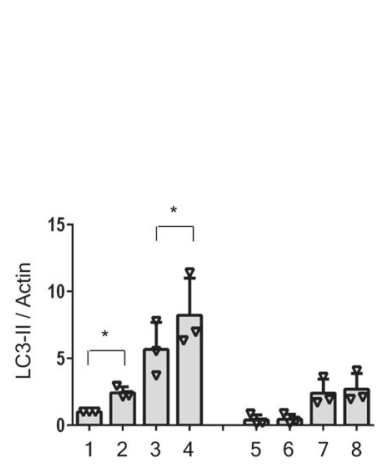

b

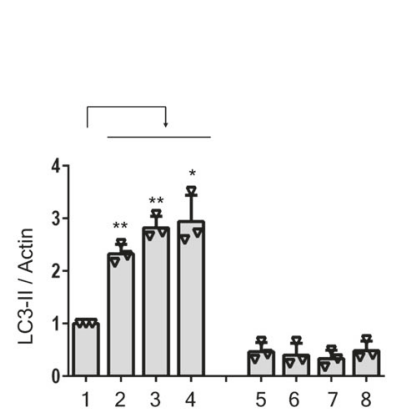

e
Fig. 7 HSV-1 induces STING-dependent autophagy. a Sting wild-type and knockout MEF cells were infected with or without HSV-1 at a multiplicity of infection (MOI) of 1 for the indicated time. Cell lysates were then immunoblotted to analyze the LC3-II conversion. Quantification of LC3-II expression levels in a is shown in the right panel (mean \pm s.d.; from three independent experiments). ${ }^{*} P<0.05$, ${ }^{* *} P<$ 0.01 . b Sting wild-type and deficient MEFs were transfected with GFP-LC3 plasmid with or without HSV-1 infection. Immunofluorescence microscopy was used to detect the formation of LC3 puncta. Scale bar: $10 \mu \mathrm{m}$. c Quantification of the numbers of LC3 puncta per cell in $\mathbf{b}$ (mean \pm s.d.; $n>100$ cells from three independent

\section{Immunofluorescence microscopy}

Cells were transfected with the indicated plasmids or cyclic dinucleotides, or infected with HSV-1 virus. The cells were fixed with $4 \%$ formaldehyde for $15 \mathrm{~min}$ at $37^{\circ} \mathrm{C}$ and permeabilized with $0.2 \%$ Triton $\mathrm{X}-100$ for $15 \mathrm{~min}$ at $4{ }^{\circ} \mathrm{C}$. Then the permeabilized cells were blocked and incubated with the indicated primary antibodies at $4{ }^{\circ} \mathrm{C}$ overnight, followed by incubation with fluorescence-conjugated secondary antibodies at room temperature for $1 \mathrm{~h}$. The images were observed using an LSM 510 Zeiss confocal microscope (Carl Zeiss Jena, Germany).

\section{GST pulldown assay}

GST and GST-LC3 proteins were expressed and purified from Escherichia coli Rosetta (DE3). Cell lysates were incubated with prepared GST or GST-LC3 protein at $4{ }^{\circ} \mathrm{C}$ overnight, and then washed three times with phosphatebuffered saline (PBS). The precipitated complex was boiled
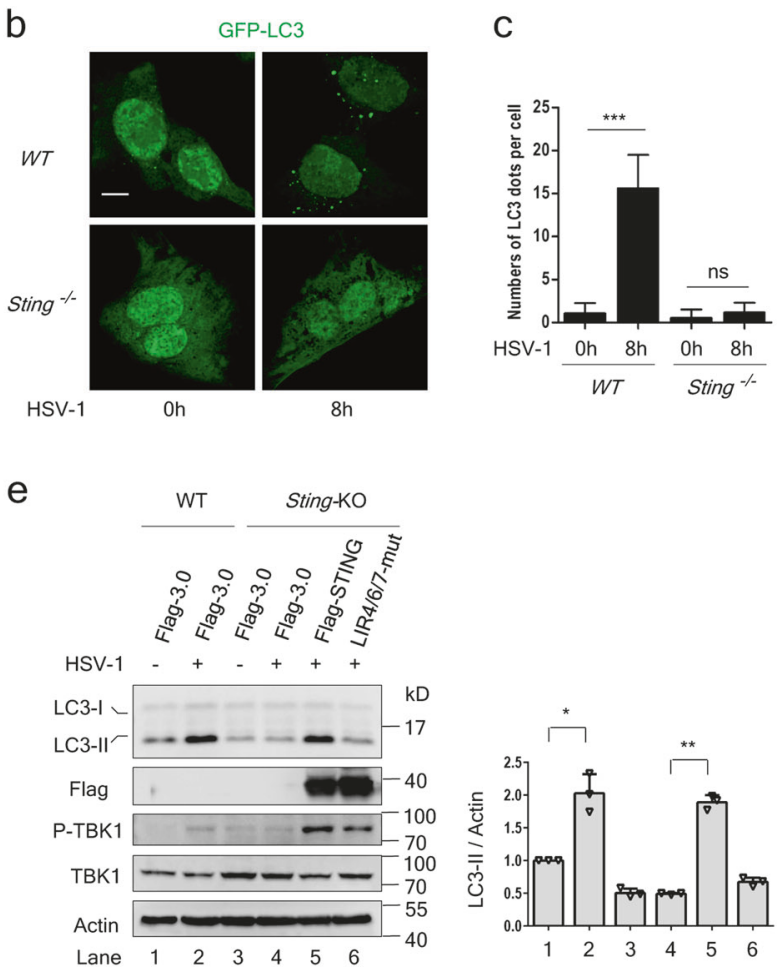

experiments). ${ }^{* *} P<0.001$. ns not significant. d Sting wild-type and knockout MEF cells were treated with or without HSV-1 or chloroquine (CQ), and autophagic flux was detected by western blotting. Quantification of LC3-II expression levels in $\mathbf{d}$ is shown in the right panel (mean \pm s.d.; from three independent experiments). ${ }^{*} P<0.05$. e Immunoblot analysis of LC3-II expression levels in Sting wild-type and knockout HeLa cells expressing the indicated plasmids with or without HSV-1 infection. Quantification of LC3-II expression levels in e is shown in the right panel (mean \pm s.d.; from three independent experiments). ${ }^{*} P<0.05,{ }^{* *} P<0.01$

for 5 min with loading buffer and subjected to SDS-PAGE and immunoblotting. The nitrocellulose membrane was stained with Ponceau $\mathrm{S}$ and then incubated with the indicated antibodies.

\section{Ubiquitination assay}

MEF cells were transfected with poly(dA:dT) at a final concentration of $1 \mu \mathrm{g} / \mathrm{ml}$, with or without Chloroquine or MG132 treatment. Then the cells were harvested and lysed with IP buffer, followed by incubation with protein $\mathrm{A}$ beads and indicated antibody at $4{ }^{\circ} \mathrm{C}$ overnight. Beads were washed three times with IP buffer and boiled for $5 \mathrm{~min}$ together with loading buffer. Prepared samples were subjected to immunoblotting.

\section{Electron microscopy}

Hela cells were transfected with Flag-vector or Flag-STING plasmids for $24 \mathrm{~h}$. The cells were washed with PB and fixed 
in $2.5 \%$ glutaraldehyde for $1 \mathrm{~h}$ at room temperature and then overnight at $4{ }^{\circ} \mathrm{C}$. After five washes with $\mathrm{PB}$, the samples were fixed with $1 \%$ osmium tetroxide containing $0.8 \%$ potassium ferricyanide on ice for $1.5 \mathrm{~h}$. Then the samples were washed with PB and water, stained with uranyl acetate, dehydrated with ethyl alcohol, and embedded in resin, which was polymerized for $24 \mathrm{~h}$ at $65^{\circ} \mathrm{C}$. Subsequently, the samples were sectioned, doubly stained with uranyl acetate and lead citrate, and observed using an electron microscope (Tecnai G2 F20 TWIN TMP).

\section{Dual-luciferase reporter assays}

Cells were transfected with plasmids carrying an IFN- $\beta$ luciferase reporter gene and pRL-TK together with empty vector or STING wild-type or mutant plasmids for $24 \mathrm{~h}$. Then the cells were collected and lysed. Subsequently, IFN$\beta$ luciferase activity was measured with the dual-luciferase reporter assay system (Promega) according to the manufacturer's protocols. Data were normalized by the ratio of firefly luciferase activity to renilla luciferase activity.

\section{Statistical analysis}

All experiments were performed in triplicate, and a twotailed Student's $t$ test was used to assess the significance of the data. A $P$ value of $<0.05$ was considered significant. All statistical calculations were carried out with Image $\mathbf{J}$ and GraphPad Prism 7 software.

Acknowledgements We are grateful to Professor Shuai Chen (Sun Yat-sen University Cancer Center, Guangzhou, China) for sharing the Flag-tagged STING, IFN- $\beta$ luciferase reporter gene and pRL-TK plasmids. We thank Professor Hongyu Deng (Institute of Biophysics, Chinese Academy of Sciences, Beijing, China) for providing HSV-1 virus. We thank Professor Pu Gao (Institute of Biophysics, Chinese Academy of Sciences, Beijing, China) for providing the structural analysis of STING LIR motifs. We would be grateful to Professor Pu Gao and Jingpeng Zhu (Institute of Biophysics, Chinese Academy of Sciences, Beijing, China) for technical help with protein purification. We thank Ms. Yinzi Ma and Mr. Pengyan Xia (Institute of Zoology, Chinese Academy of Sciences, Beijing, China) for their help with electron microscopy assays. We wish to thank Dr. Qing Zhong (University of Texas Southwestern Medical Center, Dallas, TX, USA) for his suggestions and critical reading of the manuscript. This work was supported by grants from the Special Fund for Strategic Pilot Technology Chinese Academy of Sciences (QYZDJ-SSW-SMC004), Fund for Strategic Pilot Technology Chinese Academy of Sciences (XDPB1002), the Natural Science Foundation of China (31790404), the Beijing Natural Science Foundation of China (5161002), 973 Program Projects (2015CB856303).

\section{Compliance with ethical standards}

Conflict of interest The authors declare that they have no conflict of interest.
Publisher's note: Springer Nature remains neutral with regard to jurisdictional claims in published maps and institutional affiliations.

\section{References}

1. Sun LJ, Wu JX, Du FH, Chen X, Chen ZJJ. Cyclic GMP-AMP synthase is a cytosolic DNA sensor that activates the type I interferon pathway. Science. 2013;339:786-91.

2. Wu JX, Sun LJ, Chen X, Du FH, Shi HP, Chen C, et al. Cyclic GMP-AMP is an endogenous second messenger in innate immune signaling by cytosolic DNA. Science. 2013;339:826-30.

3. Ishikawa H, Barber GN. STING is an endoplasmic reticulum adaptor that facilitates innate immune signalling. Nature. 2008;455:674-8.

4. Jin L, Waterman PM, Jonscher KR, Short CM, Reisdorph NA, Cambier JC. MPYS, a novel membrane tetraspanner, is associated with major histocompatibility complex class II and mediates transduction of apoptotic signals. Mol Cell Biol. 2008;28:5014-26.

5. Sun WX, Li Y, Chen L, Chen HH, You FP, Zhou X, et al. ERIS, an endoplasmic reticulum IFN stimulator, activates innate immune signaling through dimerization. Proc Natl Acad Sci USA. 2009;106:8653-8.

6. Zhong B, Yang Y, Li S, Wang YY, Li Y, Diao FC, et al. The adaptor protein MITA links virus-sensing receptors to IRF3 transcription factor activation. Immunity. 2008;29:538-50.

7. Saitoh T, Fujita N, Hayashi T, Takahara K, Satoh T, Lee H, et al. Atg9a controls dsDNA-driven dynamic translocation of STING and the innate immune response. Proc Natl Acad Sci USA. 2009;106:20842-6.

8. Ishikawa H, Ma Z, Barber GN. STING regulates intracellular DNA-mediated, type I interferon-dependent innate immunity. Nature. 2009;461:788-92.

9. McWhirter SM, tenOever BR, Maniatis T. Connecting mitochondria and innate immunity. Cell. 2005;122:645-7.

10. Chen Q, Sun L, Chen ZJ. Regulation and function of the cGASSTING pathway of cytosolic DNA sensing. Nat Immunol. 2016;17:1142-9.

11. Yin Q, Tian Y, Kabaleeswaran V, Jiang XM, Tu DQ, Eck MJ, et al. Cyclic di-GMP sensing via the innate immune signaling protein STING. Mol Cell. 2012;46:735-45.

12. Liu SQ, Cai X, Wu JX, Cong Q, Chen X, Li T, et al. Phosphorylation of innate immune adaptor proteins MAVS, STING, and TRIF induces IRF3 activation. Science. 2015;347:aaa2630.

13. Tanaka Y, Chen ZJJ. STING specifies IRF3 phosphorylation by TBK1 in the cytosolic DNA signaling pathway. Sci Signal. 2012;5:ra20.

14. Tsuchida T, Zou J, Saitoh T, Kumar H, Abe T, Matsuura Y, et al. The ubiquitin ligase TRIM56 regulates innate immune responses to intracellular double-stranded DNA. Immunity. 2010;33:765-76.

15. Zhang J, Hu MM, Wang YY, Shu HB. TRIM32 protein modulates type I interferon induction and cellular antiviral response by targeting MITA/STING protein for K63-linked ubiquitination. J Biol Chem. 2012;287:28646-55.

16. Zhong B, Zhang L, Lei CQ, Li Y, Mao AP, Yang Y, et al. The ubiquitin ligase RNF5 regulates antiviral responses by mediating degradation of the adaptor protein MITA. Immunity. 2009;30:397-407.

17. Qin Y, Zhou MT, Hu MM, Hu YH, Zhang J, Guo L, et al. RNF26 temporally regulates virus-triggered type I interferon induction by two distinct mechanisms. PLoS Pathog. 2014;10:e1004358.

18. Mizushima N, Ohsumi Y, Yoshimori T. Autophagosome formation in mammalian cells. Cell Struct Funct. 2002;27:421-9.

19. Deretic V, Levine B. Autophagy balances inflammation in innate immunity. Autophagy. 2018;14:243-51. 
20. Gao C, Cao W, Bao L, Zuo W, Xie G, Cai T, et al. Autophagy negatively regulates Wnt signalling by promoting Dishevelled degradation. Nat Cell Biol. 2010;12:781-90.

21. Jiao M, Ren F, Zhou L, Zhang X, Zhang L, Wen T, et al. Peroxisome proliferator-activated receptor alpha activation attenuates the inflammatory response to protect the liver from acute failure by promoting the autophagy pathway. Cell Death Dis. 2014;5:e1397.

22. Ren F, Zhang L, Zhang X, Shi H, Wen T, Bai L, et al. Inhibition of glycogen synthase kinase 3beta promotes autophagy to protect mice from acute liver failure mediated by peroxisome proliferatoractivated receptor alpha. Cell Death Dis. 2016;7:e2151.

23. Pilli M, Arko-Mensah J, Ponpuak M, Roberts E, Master S, Mandell MA, et al. TBK-1 promotes autophagy-mediated antimicrobial defense by controlling autophagosome maturation. Immunity. 2012;37:223-34.

24. Matsumoto G, Shimogori T, Hattori N, Nukina N. TBK1 controls autophagosomal engulfment of polyubiquitinated mitochondria through p62/SQSTM1 phosphorylation. Hum Mol Genet. 2015;24:4429-42.

25. Richter B, Sliter DA, Herhaus L, Stolz A, Wang C, Beli P, et al. Phosphorylation of OPTN by TBK1 enhances its binding to $\mathrm{Ub}$ chains and promotes selective autophagy of damaged mitochondria. Proc Natl Acad Sci USA. 2016;113:4039-44.

26. Wild P, Farhan H, McEwan DG, Wagner S, Rogov VV, Brady NR, et al. Phosphorylation of the autophagy receptor optineurin restricts Salmonella growth. Science. 2011;333:228-33.

27. Bhatelia K, Singh K, Prajapati P, Sripada L, Roy M, Singh R. MITA modulated autophagy flux promotes cell death in breast cancer cells. Cell Signal. 2017;35:73-83.

28. Moretti J, Roy S, Bozec D, Martinez J, Chapman JR, Ueberheide B, et al. STING senses microbial viability to orchestrate stressmediated autophagy of the endoplasmic reticulum. Cell. 2017;171:809-23.

29. Konno H, Konno K, Barber GN. Cyclic dinucleotides trigger ULK1 (ATG1) phosphorylation of STING to prevent sustained innate immune signaling. Cell. 2013;155:688-98.

30. Prabakaran T, Bodda C, Krapp C, Zhang BC, Christensen MH, Sun C, et al. Attenuation of cGAS-STING signaling is mediated by a p62/SQSTM1-dependent autophagy pathway activated by TBK1. EMBO J. 2018; 37:e97858.

31. Pankiv S, Clausen TH, Lamark T, Brech A, Bruun J-A, Outzen H, et al. p62/SQSTM1 binds directly to $\operatorname{Atg} 8 / \mathrm{LC} 3$ to facilitate degradation of ubiquitinated protein aggregates by autophagy. J Biol Chem. 2007;282:24131-45.
32. Noda NN, Ohsumi Y, Inagaki F. Atg8-family interacting motif crucial for selective autophagy. FEBS Lett. 2010;584:1379-85.

33. Jacomin AC, Samavedam S, Promponas V, Nezis IP. iLIR database: a web resource for LIR motif-containing proteins in eukaryotes. Autophagy. 2016;12:1945-53.

34. Ouyang S, Song X, Wang Y, Ru H, Shaw N, Jiang Y, et al. Structural analysis of the STING adaptor protein reveals a hydrophobic dimer interface and mode of cyclic di-GMP binding. Immunity. 2012;36:1073-86.

35. Gao P, Ascano M, Zillinger T, Wang W, Dai P, Serganov AA, et al. Structure-function analysis of STING activation by $\mathrm{c}[\mathrm{G}$ $\left.\left(2^{\prime}, 5^{\prime}\right) \mathrm{pA}\left(3^{\prime}, 5^{\prime}\right) \mathrm{p}\right]$ and targeting by antiviral DMXAA. Cell. 2013;154:748-62.

36. Shu C, Yi G, Watts T, Kao CC, Li P. Structure of STING bound to cyclic di-GMP reveals the mechanism of cyclic dinucleotide recognition by the immune system. Nat Struct Mol Biol. 2012;19:722-4.

37. Zhou C, Ma K, Gao R, Mu C, Chen L, Liu Q, et al. Regulation of mATG9 trafficking by Src- and ULK1-mediated phosphorylation in basal and starvation-induced autophagy. Cell Res. 2017;27:184-201.

38. Young ARJ, Chan EYW, Hu XW, Koch R, Crawshaw SG, High $\mathrm{S}$, et al. Starvation and ULK1-dependent cycling of mammalian Atg9 between the TGN and endosomes. J Cell Sci. 2006;119:3888-3900.

39. Puri C, Renna M, Bento CF, Moreau K, Rubinsztein DC. Diverse autophagosome membrane sources coalesce in recycling endosomes. Cell. 2013;154:1285-99.

40. Liu L, Feng D, Chen G, Chen M, Zheng Q, Song P, et al. Mitochondrial outer-membrane protein FUNDC1 mediates hypoxia-induced mitophagy in mammalian cells. Nat Cell Biol. 2012;14:177-85.

41. Watson RO, Manzanillo PS, Cox JS. Extracellular M. tuberculosis DNA targets bacteria for autophagy by activating the host DNAsensing pathway. Cell. 2012;150:803-15.

42. Lim J, Murthy A. Controlling inflammation by selective autophagy. Cell Death Differ. 2018;25:825-7.

43. Cheng J, Liao Y, Xiao L, Wu R, Zhao S, Chen H, et al. Autophagy regulates MAVS signaling activation in a phosphorylationdependent manner in microglia. Cell Death Differ. 2017;24:27687.

44. Fang R, Wang C, Jiang Q, Lv M, Gao P, Yu X, et al. NEMOIKKbeta are essential for IRF3 and NF-kappaB activation in the cGAS-STING pathway. J Immunol. 2017;199:3222-33. 